\title{
FACIAL FEATURE EXTRACTION USING GENETIC ALGORITHM
}

\author{
By \\ NETHRIE NITHIANANDAN \\ Bachelor of Science \\ Karnataka Regional Engineering College \\ Surathkal, India \\ 1999
}
Submitted to the Faculty of the
Graduate College of the Oklahoma State University in partial fulfillment of the requirements for the Degree of MASTER OF SCIENCE May, 2002.




\section{FACIAL FEATURE EXTRACTION USING GENETIC ALGORITHM}

Thesis Approved:

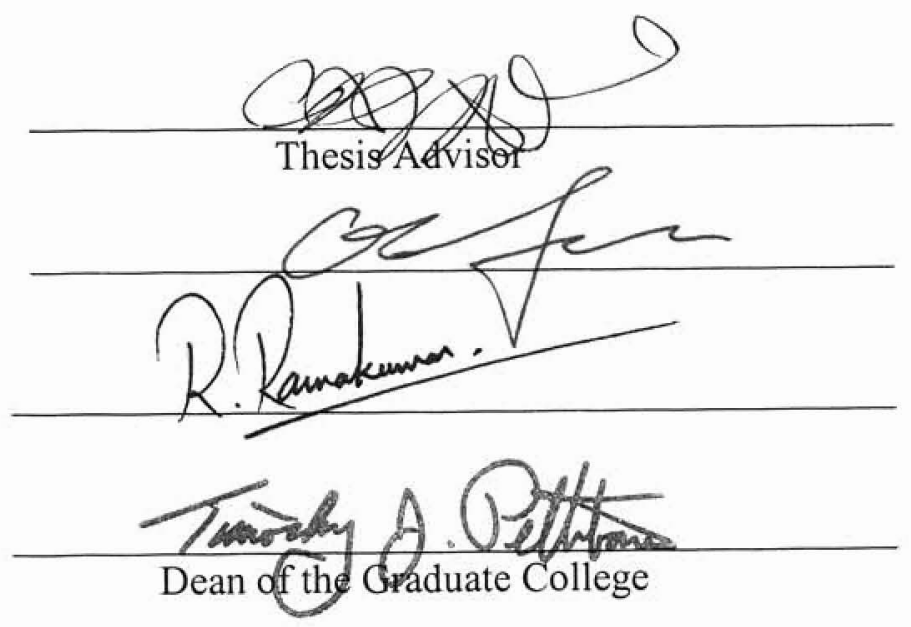




\section{PREFACE}

Facial feature extraction plays an important role in many applications such as low bit video coding, human face recognition and access control systems. An automatic feature extraction method is presented in this Thesis. In the preprocessing stage face is approximated to the ellipsoid and a genetic algorithm is used to search for the ellipse region in the image. In the feature extraction stage, the located face is converted to the edge map. Facial features such as the eyes, nose and mouth are extracted based on the edge density distribution. Genetic algorithm is used to search for the template that has the highest edge density in the sub regions.

Simulation results show that the proposed algorithm is able to successfully extract the facial features from different images under different environments and even in the presence of certain amount of artificial noise. 


\section{ACKNOWLEDGEMENTS}

The advice, guidance, support, encouragement and understanding of my learned professors and a few others, enabled me to enrich my studies and research here, in the department of Electrical and Computer Engineering at Oklahoma State University, and complete this Thesis. I wish to express my gratitude to all of them. I should first thank my advisor Professor Gary G. Yen, who was instrumental in introducing me to the problems of facial feature extraction. He devoted his most precious time in guiding me throughout my studies and how to approach and solve the problems. I wish to also thank Haiming Lu and others in the Intelligent Systems and Control Laboratory for their useful suggestions, at times. I wish to place on record the valuable advice given by Professors Dr. Ramakumar and Dr. Fun of the advisory committee. I wish to thank them for the time they have devoted and for all the invaluable feedback and suggestions, they have given me, about my research. I am also thankful to those for supplying the mask images for the facial feature extraction experiments described in this Thesis.

Finally I would like to thank my parents and siblings for always praying for my success. 


\section{TABLE OF CONTENTS}

Chapter

I INTRODUCTION 1

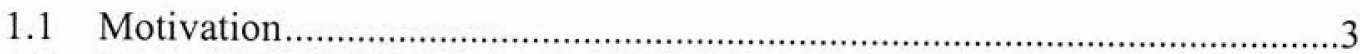

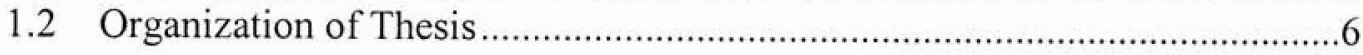

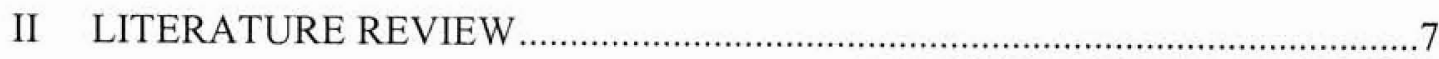

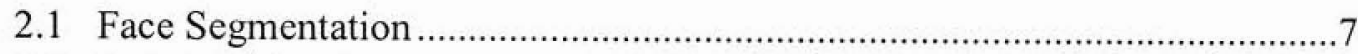

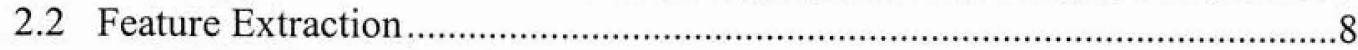

2.2.1 Feature based approach................................................................

2.2.2 Appearance based approach........................................................10

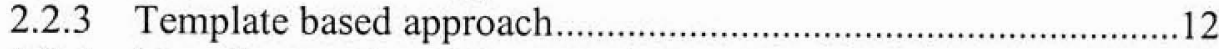

2.2.4 Neural network and fuzzy logic based approach .........................13

2.2.5 Genetic algorithm based approach.............................................16

2.2.5.1 Genetic algorithm: an overview.....................................16

2.2.5.2 Facial feature extraction using GA ……………….........19

2.2.6 Other approaches .....................................................................19

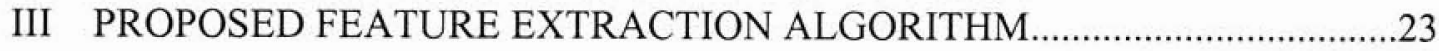

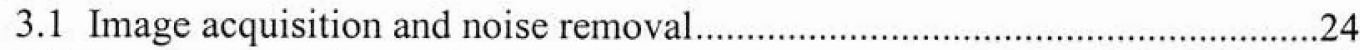

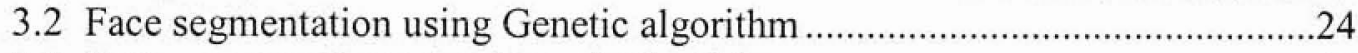

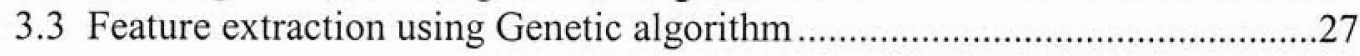

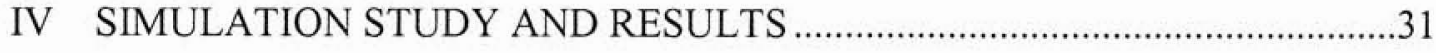

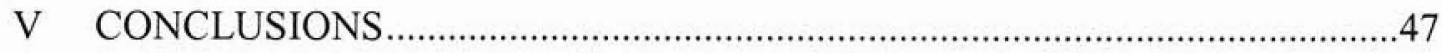

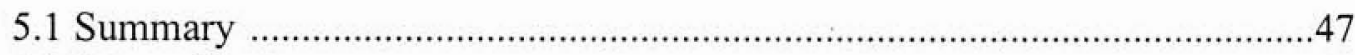

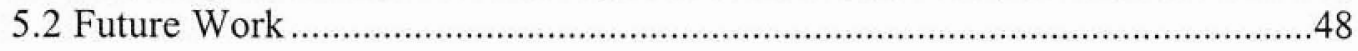

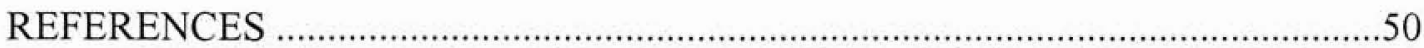




\section{LIST OF TABLES}

Table

Page

4.1 GA parameters. .32 


\section{LIST OF FIGURES}

Figure $\quad$ Page

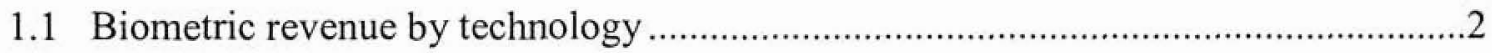

2.1 Automatic feature extraction scheme for model based coding ..................................10

2.2 Left eye masks obtained from PCA learning with only left eye images .....................11

2.3 Right eye masks obtained from PCA learning with only right eye images .................11

2.4 Nose masks obtained from PCA learning with only nose images ..............................11

2.5 Mouth masks obtained from PCA learning with only mouth images.........................12

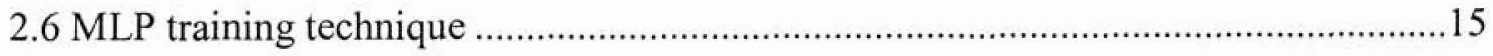

2.7 Training an MLP on the out put of the Kahonen network .........................................15

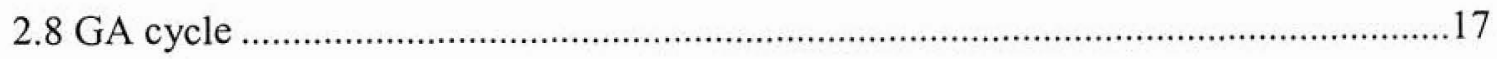

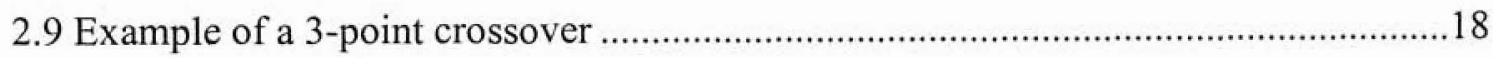

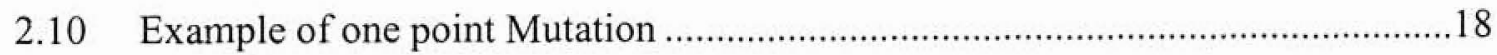

2.11 Simplified (a) eye template and (b) mouth template ….......................................20

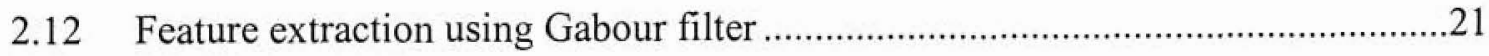

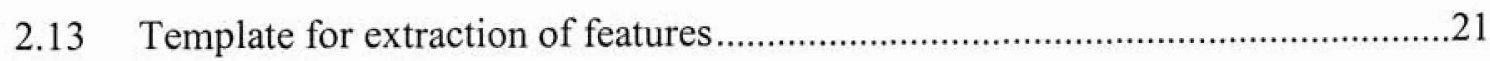

2.14 (a) y-projection (b) preprocessed face (c) $x$-projection .....................................22

3.1 Block diagram of the proposed feature extraction method ..................................23

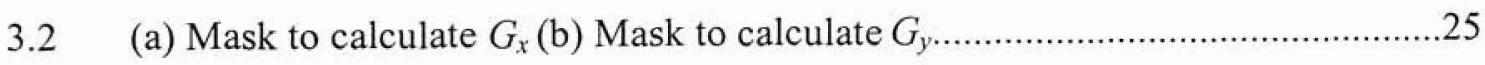

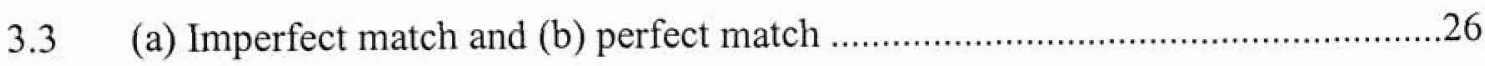




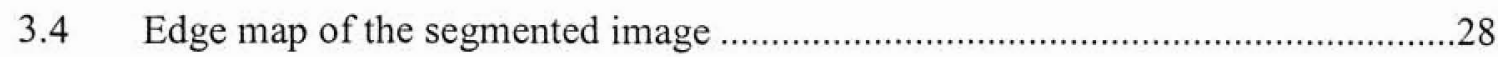

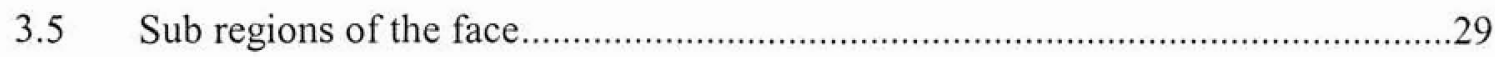

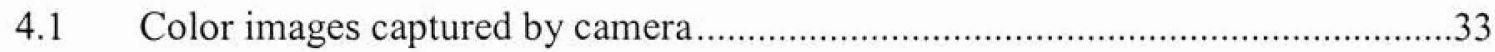

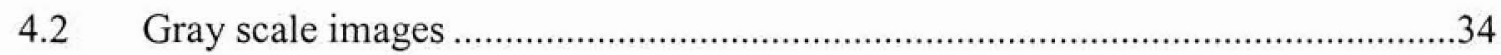

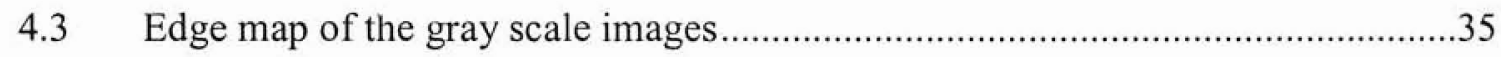

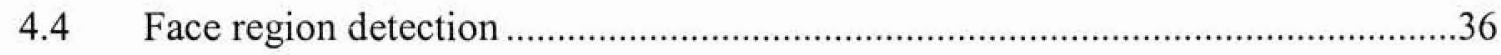

4.5 Simulation results of images with normal background..........................................37

4.6 Simulation results of images with complex background …...................................38

4.7 Simulation results of images with different expressions ......................................39

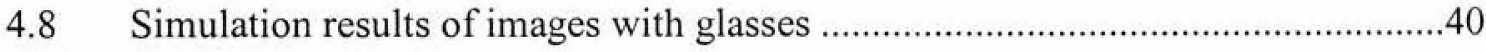

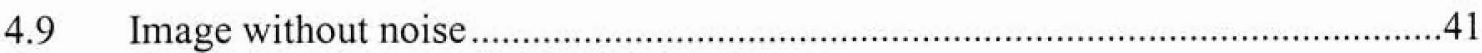

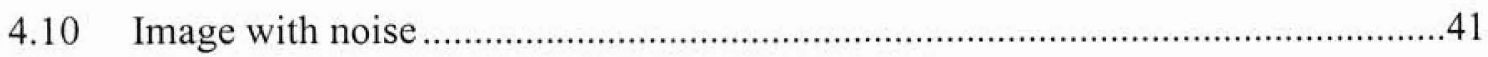

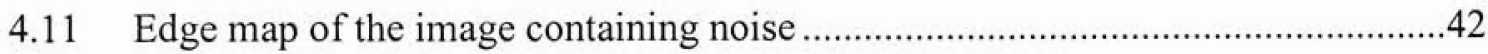

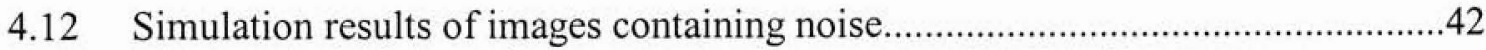

4.13 Simulation results of images oriented at an angle ................................................43

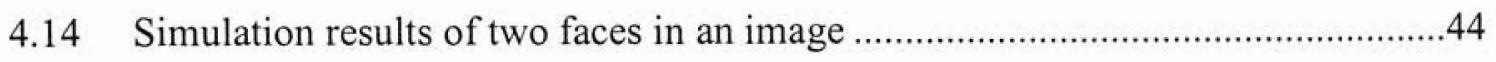

4.15 Simulation results of two faces in an image ......................................................

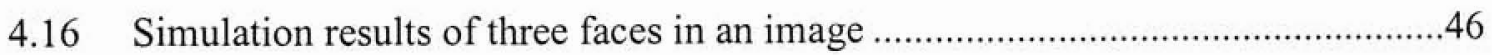

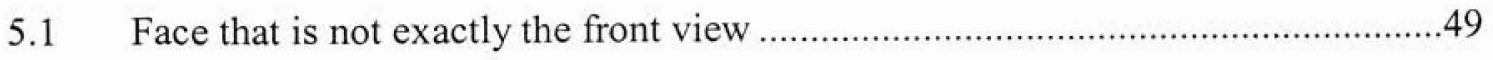




\section{CHAPTER I}

\section{INTRODUCTION}

Modern multimedia advancements have inevitably led to the development of images, audio and video applicaions in medicine, remote sensing, security, entertainment and education. Effective image feature extraction is an important task for multimedia information management. In order to efficiently retrieve a particular image from a large data base, it shoud be either described by some keywords or automatically extracted visual features. However, in the case of a large image data base it will not be efficient to describe an image with keywords, as this not only requires extensive labour but also due to the fact that there are insufficient amount of keywords to effectively characterize the contents of the image. Therefore, automatic feature extraction and indexing is in great demand [1]. In general, the objective of the feature extraction phase is to reduce the dimension of the raw image while at the same time retaining as many salient features as possible.

Emerging information technologies such as biometrics, medicine and geographical information systems have greatly benefitted by the application of efficient feature extraction techniques. 
Biometrics identification is a broad category of technologies based on some biometric measures of humans. These quanititative measures are used to identify an individual's physical characteristics such as the fingerprints, retinal scans, facial features, etc [2]. Feature extraction of biometrics is considered to be a key component in many applications. These applications include, but not limited to, security (e.g. log-in control, physical access authentification, surveillance), law enforcement (mug shot albums, criminology) and commercial applications (passports, credit cards, driver's licenses) [2,3]. Figure 1.1 shows biometric revenue in 1999 by different technologies used in various industries [4]. This chart shows the growing importance of the applications of biometric data. Even though revenue from retina-scan accounted for only 2 percent, it is believed that eye scanning and finger printing are widely used in the law enforcement sector.

1999 Biometric Revenue By Technology

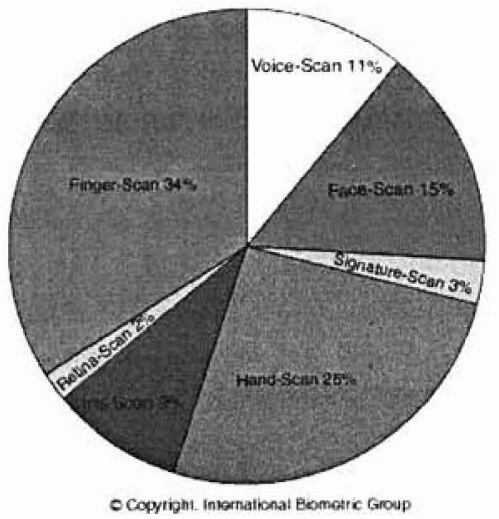

Total 1999 Revenue: S58.4 Million

Projected 2003 Revenue: $\$ 594.0$ Million

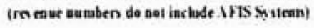

Figure 1.1 Biometric revenue by technology 
Feature extraction is used in the case of Geographical Information Systems to detect faults and extract some features in geoscientific datasets. For the purpose of analysis, orientation information of features such as joints, cracks and dykes of all scales are extracted from the groscientific datasets. These features had to be extracted from other geological featues contained in the data in the form of noise [5]. Information on potential overhead line defects is required to repair them acculrately and promtly. The electricity companies capture pictures of the overhead lines and applies appropriate feature extraction algorithm to detect the fault, if any [6].

Feature extraction in medical images is popularly used for diagnosis. The main cause of blindness in the working age population is diabetic retinopathy. Feature extraction is used to detect the main abnormal features from diabetic retinopathy such as the optic disc, blood vessels and exudates [7]. Feature extraction has also been used in breast cancer diagonosis based on mammograms. Lobulation and microlobulation of nodules are two important features in the ACR (American College of Radiology) lexicon [8]. Applicationspecific feature extraction algorithms have been developed to extract these features.

\subsection{Motivation}

Facial feature extraction plays an important role in applications such as low bit video coding, human face recognition and access control systems $[9,10]$. State-of-the-art multimedia teleconferencing systems are based on digital video coding and transmission of television pictures. To use the low bandwidth digital networks for this purpose, 
significant compression of data is required. Images can be considered as having structural features such as contours and regions. These images have been exploited to encode images at low bit rates, while retaining sufficient visible structures in the reconstruction so as to maintain an acceptable level of quality [10-12].

Smart cards are widely used for security access to public buildings, military installations and computer networks. This technology is considered to be the most effective, efficient, and economic by means of ensuring security access. These cards store unique biometric data in the memory. The biometric data that has been chosen are data relating to fingerprints, signature, retinal pattern, voice recognition and faces [4]. Feature analysis is perhaps the most widely utilized approach in face recognition.

According to "USA today" [13], the need for enhanced recognition technology to support surveillance is becoming very important. Surveillance cameras are fixed on the subway, city centers, parks, shopping malls, buses, roadways and even in the historic rural villages in Britain. It is proven that this kind of security measures will not only reduce the percentage of burglary and shop lifting but also will help to identify the culprits on-thefly. It is also predicted that thousands of such surveillance systems will be installed across the USA at airports, train stations, stadiums, public monuments, ATM machines and even in private business as a result of September 11 th attack on the World Trade Center. 
Developing a computational model for face recognition is quite challenging, if not impossible, because faces are complex and multidimensional [3]. During the last 20 years extensive research has been conducted by psychophysicists, neuroscientists and engineers on various aspects of face recognition by trained humans and machines [14]. In the early and mid 1970's, classical pattern classification techniques, which use the measured attributes between features and face profiles, were used for face recognition with very little success. During the 1980's, the work on face recognition was dormant [1]. But for the last 5 years or more, attention is re-focused on segmentation and location of the face in a given image and extraction of features such as eyes, mouth etc [3].

The process of face recognition consists of the following steps:

- sample capture,

- feature extraction,

- template comparison, and

- matching.

The samples are captured generally using a digital camera where snapshots are taken from one's face. The next step is to extract the distinctive features from the captured sample faces. This will result in creation of different templates, which are much smaller than the image from which they are drawn. Identification and verification are performed next by comparing the new templates with the existing prototypes in the database [15]. 
One of the important tasks in the face recognition process has been studied in this thesis. The goal of this project is to investigate feature extraction of facial images using genetic algorithm, a population-based optimization algorithm that has emerged in the spirit of natural survival of the fittest.

\subsection{Organization of Thesis}

The thesis is structured as follows. In Chapter II, a literature review of various feature extraction methods are presented. Chapter III presents the proposed genetic-algorithmbased feature extraction and its application in facial feature extraction. Simulation results are used to validate the effectiveness of the proposed approach in Chapter IV. Chapter V documents the conclusions and outlines potential future research directions. 


\section{CHAPTER II}

\section{LITERATURE REVIEW}

The main process of feature detection would be to remove the background information that forms a part of the pictures. Image segmentation is the most critical part in an image processing application. It refers to the grouping of different parts of the image that has similar image characteristics. Subsequent interpretation tasks such as feature extraction, object recognition and classification depend on the effective segmentation technique implemented [16].

\subsection{Face Segmentation}

Some common techniques are used in the case of face detection. The face region is detected using skin color information. Normalized RGB (Red, Green and Blue) color model and the HSV (Hue, Saturation and Vertical) color model are often used. Faces were detected using fuzzy pattern matching with the Skin and Hair color distribution [17]. $\mathrm{YCbCr}(\mathrm{Y}$ - luminance, $\mathrm{Cb}$ and $\mathrm{Cr}$-Chrominance) color space is also used to exploit the skin color information, which is followed by simple morphological operations such as dilation and erosion [18]. One open problem of color - information - based face location is that, when there are non-face skin color regions such as hands and shoulders in the background, it is difficult to locate the face solely by the skin color information. To avoid 
this problem, a method that approximates the face region to an ellipsoid is employed. This method can detect face regions from complex background and also it does not impose any constraints on the face size and position [19].

\subsection{Feature Extraction}

Feature extraction can be performed using various mathematical models, image processing techniques and computational intelligent tools such as neural networks or fuzzy logic. They are generally classified into three categories, namely, feature based, appearance based, and template-based approaches.

\subsubsection{Feature based approach}

In the feature-based approach, image feature is extracted based on geometric relationships such as position, width of the eyes, eyebrow thickness and arches, face breadth, etc. The features are extracted from the vertical and horizontal integral projections of the original image. It is assumed that all faces have two eyes, nose and a mouth with similar layout. The horizontal edge map is used to extract the left and right boundaries of the face and the nose, while the vertical edge map is used to extract the eyes, mouth and nose base [20]. The peaks and valleys of the horizontal and vertical projections are analyzed with respect to a threshold value to detect and extract the positions of the features. In a method discussed in [21], features are extracted in two stages. Regions near eyes, eyebrows and mouth have many long horizontal edges. 
Regions where the average density of the horizontal edges is high compared to the rest of the face regions are identified in the first stage. In the second stage the integral projection approach is applied to those regions to detect the feature positions. To obtain reliable performance both magnitude and sign of the image gradient are obtained with respect to a threshold value calculated using the image gradient. These extracted feature positions are compared to the relational face model that describes the geometrical relationship between the features to verify whether the face candidate is a human face. The geometric relationship of the eyes and the mouth in the human face is assumed to be approximately the same. In the methods discussed in [20] and [21], selection of the threshold value plays a key role in the extraction.

Chuang et al in [12] have developed a feature-based approach for model based coding. The automatic feature extraction scheme adopted in [12] is shown in Figure 2.1. Histogram equalization and edge detection methods were applied and the edge points were retained. The edge map of the face region is divided into three equal areas in vertical direction and vertical integral projection was employed on the edge map to search for the feature elements. Using threshold operation at each facial area, and applying local threshold, every control point of the facial features such as the eye and mouth was obtained [12]. In order to obtain good results, it is assumed that the background is smooth and its luminance is different from the head and the clothes of the person, the gray level of the person's hair is lower than that of the persons face and the person does not wear glasses. Ham et al [22] discuss a knowledge based algorithm where the features are defined by the widths of the eyes and mouth and the distances from nose 
to mouth and from eyes to nose [22]. Even though this method does not require any human interaction for the selection of threshold values, it assumes the horizontal projection value for the eye is the maximum and the background is dark [22].

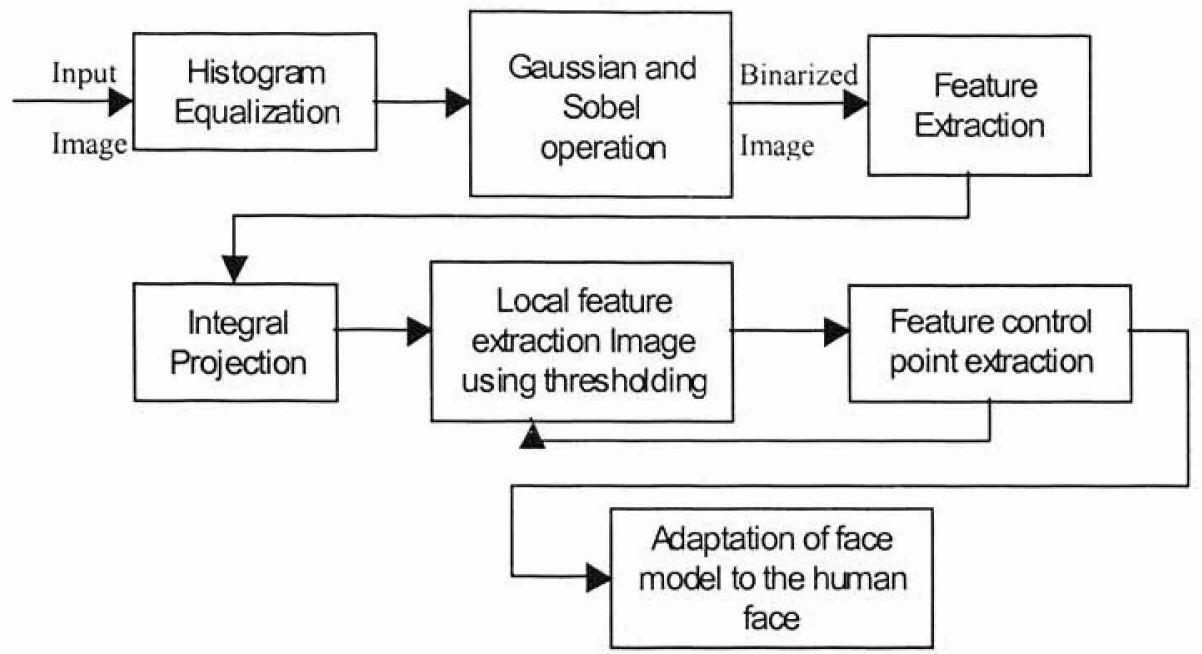

Figure 2.1 Automatic feature extraction scheme for model based coding

\subsubsection{Appearance based approach}

The principal component analysis (PCA) is a powerful statistical approach to extracting facial features for recognition [23]. This approach transforms face images into small set of characteristic feature images called "Eigenfaces", which are principal components of the initial training set of face images [24,25]. Eigenfaces are nothing but a set of orthonormal basis vectors. Each of these basis vectors can be displayed as a ghostly face, therefore it is referred to as an eigenface $[24,26]$. Concept of eigenfaces can be extended to eigenfeatures, such as eigeneye, eigenmouth and eigennose. These eigenfeatures are used for the detection of features such as eyes, mouth and nose [3]. In a case where there 
are $\mathrm{N}$ faces in the training set and $\mathrm{Q}$ features per face it is possible to obtain $\mathrm{N} \times \mathrm{Q}$ feature points. Considering the number of PCA masks to be K, the whole face database is represented by $\mathrm{N} \times \mathrm{Q} \times \mathrm{K}$ values. Using the $\mathrm{N}$ training images, the average matrix is obtained. The covariance matrix is calculated. Finally the eigenvectors and the associated eigenvalues are evaluated from the covariance matrix $[10,23,25,27]$. Figures 2.2 through 2.5 show the first three principal components of features: left eye, right eye, nose and mouth calculated using the above method respectively. In the eigenfeature representation the equivalent distance from the feature space is effectively used for detection of features. In case of a new input image, distance from the feature space is computed at each pixel and the minimum of the distance map is considered as the best match [28]. Eigenface approach assumes that the data are in low dimension.
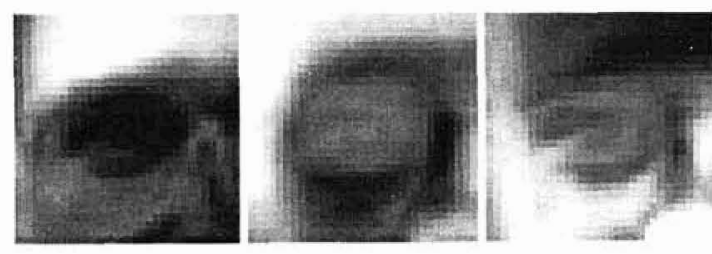

Figure 2.2 Left eye masks obtained from PCA learning with only left eye images
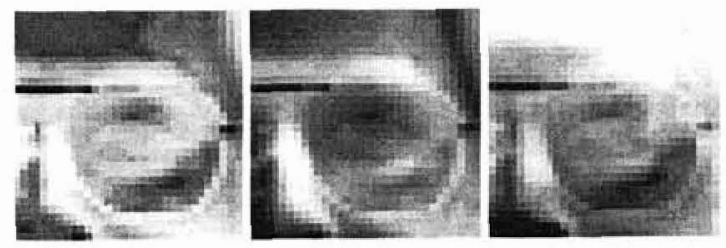

Figure 2.3 Right eye masks obtained from PCA learning with only right eye images
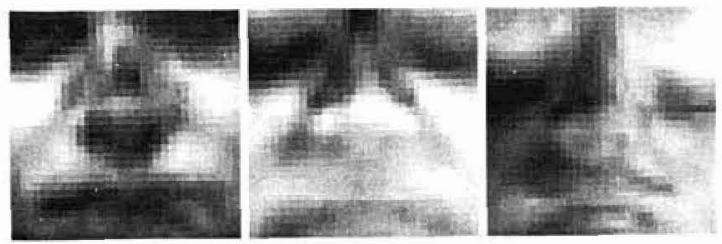

Figure 2.4 Nose masks obtained from PCA learning with only nose images 

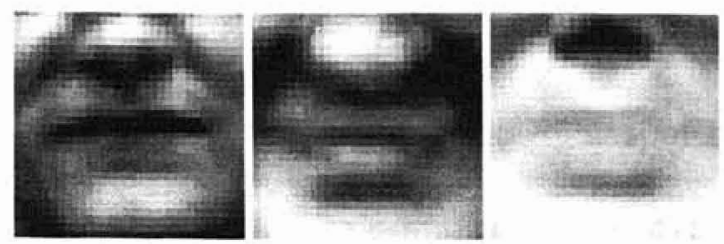

Figure 2.5 Mouth masks obtained from PCA learning with only mouth images

\subsubsection{Template based approach}

Template based approach is one of the conventional techniques which is still being popularly used in various applications. In early 80 's Baron proposed a simple feature extraction based on template matching [29]. He used 16 different eye templates to match with the sub images from the input image. A correlation value greater than 0.8 for any eye template indicates the location of the eye. The whole image is used to search for a particular feature. Also there are chances where the particular shape of an eye may have been missed in the collection of templates [29]. The principle developed in [29] is adopted in [30] but the matching function is based on pixel density distribution, which is shown below,

$$
D(x, y)=\sum_{i} \sum_{j}\{G(x+i, y+j)-T(i, j)\}^{2},
$$

where $D(x, y)$ represents the degree of matching between the gray scale image $G(x, y)$ and the template $T(x, y)$ at the location of $(x, y)$ in the input image. $(i, j)$ denote the coordinates of the template. The feature is detected when the value of $D$ is minimum. 
The template model can be represented by cost function or energy function for different features [9,31]. Lau et al [9] propose an energy function, which consists of central weighting, mean crossing, intensity, edge response, light spot and eye white function (exists only when the eyes are open). The point under the eye should have low value according to the design function. This energy function is developed only to locate irises. Hung in [31] developed different cost functions for different regions such as the eye region and the mouth region. Genetic algorithm is used to search for the best match. Even though the above algorithm has shown promising results, it has not dealt with some particular conditions such as a person wearing spectacles or person with either beard or mustache.

\subsubsection{Neural network and fuzzy logic based approach}

Artificial neural networks enable solution to be found to problems where algorithmic methods are too computationally intensive or algorithmic methods do not exist. The applications of artificial neural networks in detecting the features on low-resolution images were not very successful [32,33]. Hilen et al [32] discussed a multi layer perceptron network (MLP) using back propagation training rule. The resolution of initial database of images was 512 by 512 . For the MLP to process the entire image, it had to undergo sub sampling and windowing. Once the sub sampling is carried out, select a suitable window, which is large enough to enclose all the pixels, which account for the eye. The MLP consists of an input layer, $x$ times $y$ units, a hidden layer and an output layer of one unit. The MLP is trained such that the output is one only when the eye is 
located. This method was unable to locate eye when the image contained points similar to the eye in the hair region or in the cloth region [32]. Therefore, to rectify these kinds of problems, high-resolution images were used. Hutchinson et al [33] discusse two different methods of feature extraction; one using MLP and the other using a combination of Kohonen network and MLP. In the first method, the input layer is of size 16 by 16 units, which corresponds to the window size of the selected region in the image. Two different sizes of hidden layer, 16 and 32, and the output layer of size one unit are used. There was no direct connection between the input and the output layer and thus the network does not have the ability to learn that the input data is two-dimensional. The training technique is shown in Figure 2.6. The second method is the combination of Kohonen and MLP. The Kohonen has an input layer of size 16 by 16 units corresponding to the window size and a two-dimensional output layer of size 10 by 10 units. The MLP is used for decoding the output of Kohonen network as shown in Figure 2.7. Even though this method had produced better results than the method discussed in [33], to produce accurate and robust results using neural network systems, it will be better if we could include hierarchical systems incorporating both local (feature information) and global (face) image data.

To improve the speed of the matching process, a fuzzy neural network is proposed by $\mathrm{Yu}$ et al [34]. Fuzzy neural network can process ambiguous or imprecise data and can learn from training examples. The templates that fall into the fuzzy class boundary are selected, and the multiple super class neurons were used to find the best matched template. Even though the searching rate of fuzzy neural network is faster than the conventional techniques it still depends on the fuzzy class boundaries [34]. Label graph templates are 
relational data structures that enable an object model to carry various types of image information. The Gabour wavelet filters are used to extract the low dimension features from the image to label the vertices of the graph template. A back propagation network is employed to extract the best template model from the training set of templates [35].

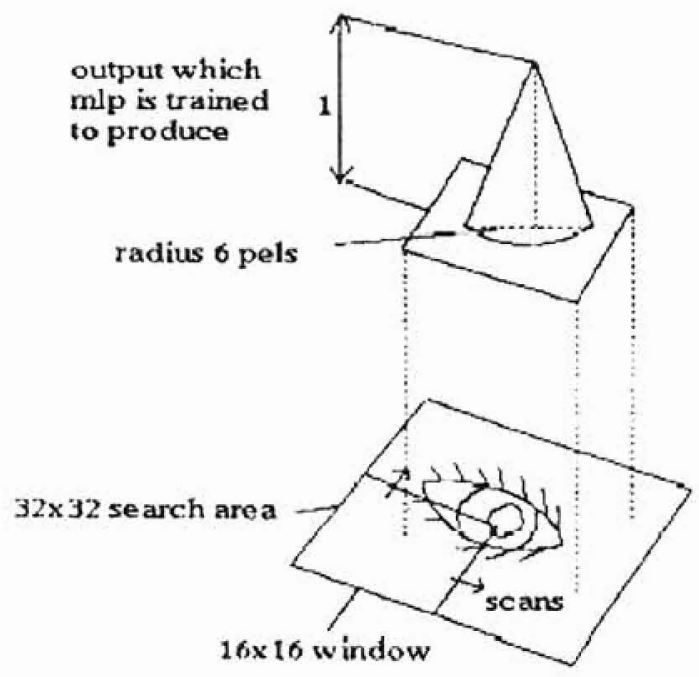

Figure 2.6 MLP training technique

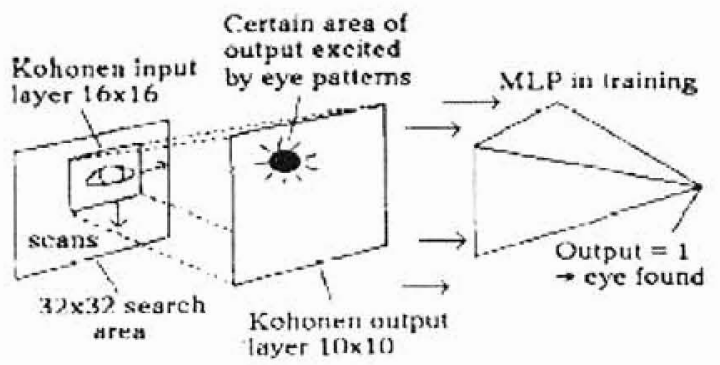

Figure 2.7 Training an MLP on the output of the Kohonen network 


\subsubsection{Genetic algorithm based approach}

\subsubsection{Genetic Algorithm: an overview}

Genetic algorithms (GAs) are search algorithms based on the mechanics of natural selection and natural genetics [36]. The basic principle of GA was first proposed by Holland [36], based on the concept of Darwin's "survival of the fittest". It assumes that the potential solution of a problem is represented by parameters called the genes of the "chromosomes" (a string of ones and zeros) [37,38]. The population comprises of a group of chromosomes from which candidates can be selected for the solution of a problem [37]. In every generation a new set of artificial creatures (strings) is created using a kind of "natural selection" [39]. It is important to know the difference between the genotype and the phenotype. If a population is constructed as an array of individuals, the artificial chromosomes are called the genotype and the decoded parameters are called the phenotype. A simple genetic algorithm that yields good results in practical problems is composed of three operators namely, reproduction, crossover and mutation. Reproduction is a process where the individual strings are copied according to their objective function [36]. Parent selection emulates the survival of the fittest mechanism in nature. It is expected that a better fit chromosome reproduces a higher number of offspring and thus has a higher chance of surviving in the subsequent generations. On the other hand, betterfit chromosomes will replace ill-fit chromosomes whose fitness values are small. Therefore the quality of the chromosome in the population will become better and better over generations in term of chosen fitness function. The "Roulette Wheel" selection scheme is one of the most commonly used selection techniques $[37,38]$ The strings with 
high fitness will have a high probability of contributing one or more offspring in the next generation [38]. Crossover exchanges subparts of two parent chromosomes to produce offspring that contain some part of both parent's genetic sequences. Single and multipoint crossover defines where the chromosome can be recombined. In a single point crossover, the crossover point is randomly selected and the portions of the two chromosomes beyond this point are exchanged to form two offspring. In a multipoint crossover two or more points are chosen at random without duplication [37]. The probability term $p_{c}$ is set to determine crossover operation rate. [36-38]. Mutation randomly changes the bit value of some locations in the chromosome [39]. This operation occurs occasionally, usually with a small probability of $p_{m}$ [37]. It is observed that for a small population $(<100)$ the crossover probability of 0.6 and mutation probability of 0.01 and for large population $(>100)$ the crossover probability of 0.8 and mutation probability 0.001 yields good results [36]. A typical GA cycle is shown in Figure 2.8. Figure 2.9 is an example of a 3-point crossover. Three arrows point at the points at which the crossover takes place. Figure 2.10 is a one point mutation where the 1 in the fourth position is converted to 0 .

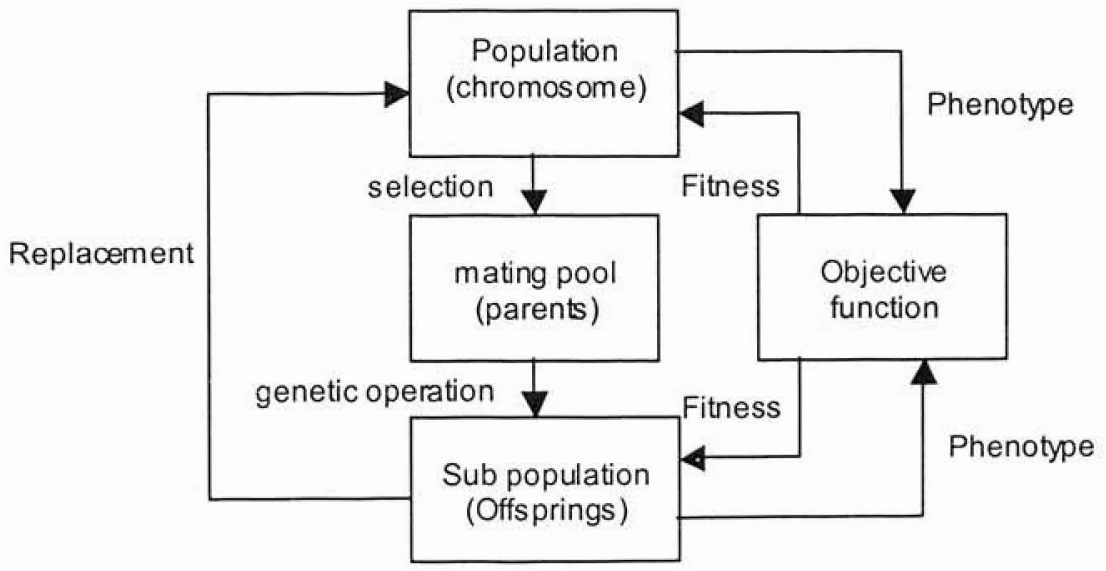

Figure 2.8 GA cycle 

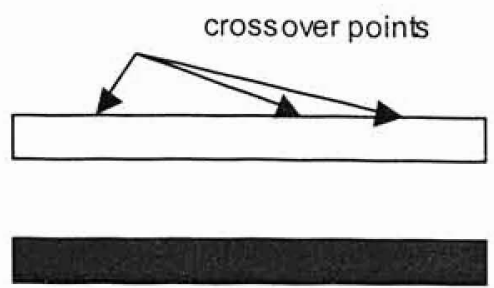

Parents
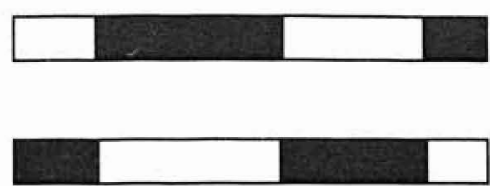

Offsprings

Figure 2.9 Example of a 3-point crossover

\begin{tabular}{|c|c|c|c|c|c|c|c|c|}
\hline original chromosome & 1 & 1 & 0 & 0 & 1 & 0 & 1 & 0 \\
\hline New Chromosome & 1 & 1 & 0 & 1 & 1 & 0 & 1 & 0 \\
\hline
\end{tabular}

Figure 2.10 Example of one point mutation

GA is different from the traditional search methods in the following ways.

- GA works with a coding of parameter sets and not with the parameters themselves.

- GA searches from a population of points, not a single point.

- GA uses objective function information, not derivative or other auxiliary information.

- GA uses probabilistic transition rules, not deterministic rules.

- The result is a population of solutions instead of an individual solution. 


\subsubsection{Facial feature extraction using GA}

Lin et al discuss an automatic feature extraction method using genetic algorithm. Single feature template is designed to extract the features [9]. Predefined cost functions will measure the fitness of the chromosome in the search space. Cost function consists of mean crossing function and central weighting function. The face is divided into sub regions and the features such as eyes, nose and mouth are searched in their respective sub regions. The best matching templates are obtained as the feature points of the face [9].

By examining a large set of face images, it has been observed that the intensity values in facial feature regions form a deep valley. Human eye regions are detected by testing all the valley regions in the image. The pairs of eye candidate are selected using genetic algorithm. The fitness function of the GA is defined by the eigenface technique discussed in Subsection 2.2.2 [24]. In order for the genetic algorithm to perform well, the population is expected to be large. That is the number of eye candidates should be large [40].

\subsubsection{Other approaches}

The deformable templates are flexible templates constructed with a - priori knowledge of shape and size of the different features. The templates can change their size and shape so that they can match the data [41]. Each of the templates has been evaluated by an energy function, defined in terms of peaks and valleys of the image intensity, edges and the intensity itself. The minimum value of the energy function corresponds to the best fit 
with the image. This method works well in detection of eyes and mouth despite variations in tilt, scale, rotation of head. However, modeling of the nostril, eyebrow and face was always a difficult task $[41,42]$. The simplified deformable template models of the eye and mouth are shown in Figure 2.11.

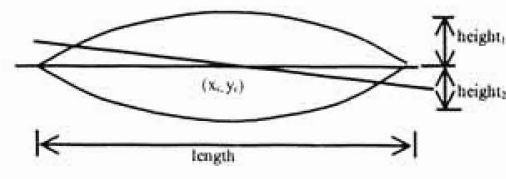

(a)

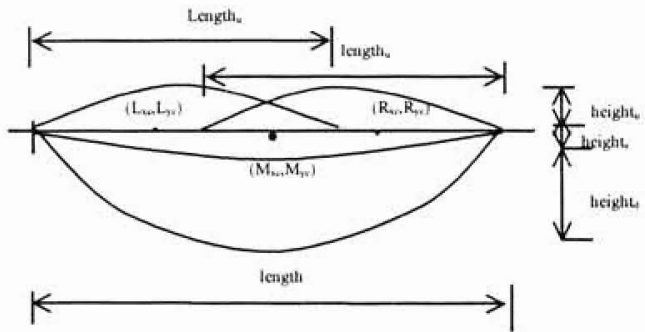

(b)

Figure 2.11 Simplified (a) eye template and (b) mouth template

Another simple method for extraction of features is using Gabour filter method as shown in Figure 2.12 [43]. This method helps to detect features when the extraction of features need not necessarily be accurate. The Gabour filter is applied on the image and then the 2D Gaussian filter multiplies it. The peaks of the image are the features points [43]. A novel valley detector filter is proposed in [44]. It has been observed that the luminance values form a deep valley in the facial feature regions including eyes, nose and mouth. It is said that the proposed filter was able to detect edges more accurately than any other filters including the Sobel edge detector [45] or a morphological filter [45]. Specifically the edges of eyes and mouth are prominent. The energy function consists of valley energy, edge magnitude and image intensity. The features are finally extracted where the sum of the energy function is maximum over a certain template region [44]. The template used for extraction is shown in Figure 2.13. $E_{x}$ and $E_{y}$ are the horizontal and vertical 
distances and $M_{y}$ is the distance between the eyes and mouth. Valley and Edge energy with respect to the energy function is calculated in the upper box and the intensity is calculated in the lower box.
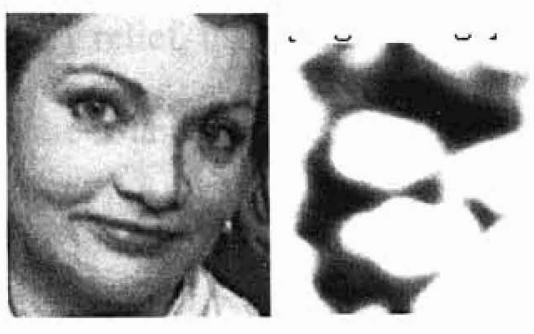

Original Image Gabour filtered
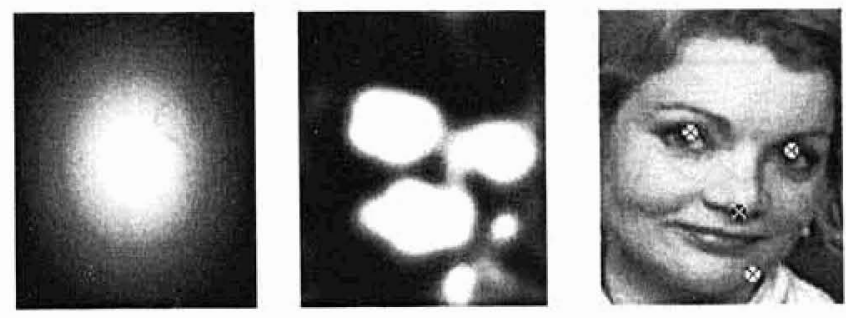

2-D Gaussian

Gabour*Gauss

Located Feature Image points

Figure 2.12 Feature extraction using Gabour filter

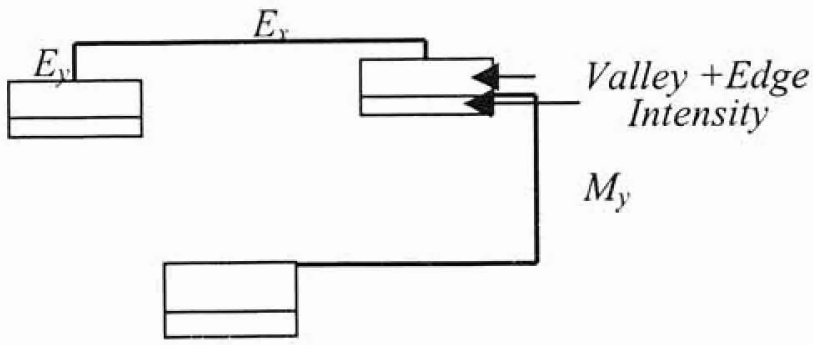

Figure 2.13 Template for extraction of features 
Feature points can be extracted with the knowledge of color information. In the intensity image, eyes and mouth differ from the rest of the face because of their lower brightness. The color of the pupil and the light red color lips are the reason for lower brightness. The $x$ and $y$ projections of gray level relief are shown in Figure 2.14. The mean gray level of each row is used to compute the $y$ relief, using the minimum values of $y$ relief, $x$ relief is computed. With help of both the $x$ and $y$ projections, the positions of the features are obtained [46].

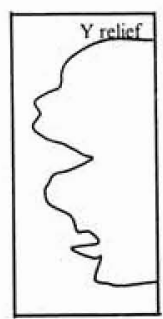

(a)

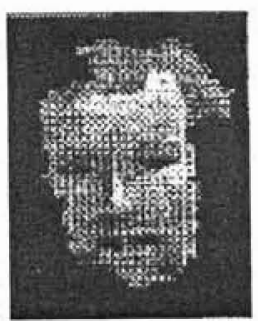

(b)

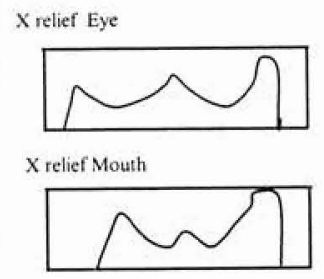

(c)

Figure 2.14 (a) $y$-projection (b) preprocessed face (c) $x$ - projection 


\section{CHAPTER III}

\section{PROPOSED FEATURE EXTRACTION ALGORITHM}

In this chapter we will discussion to the proposed feature extraction algorithm. The head and shoulder images of the front view of the images are considered for the experiment. Additionally, the images were taken when the person is not moving. Figure 3.1 presents an overview of the process involved in extracting the facial features.

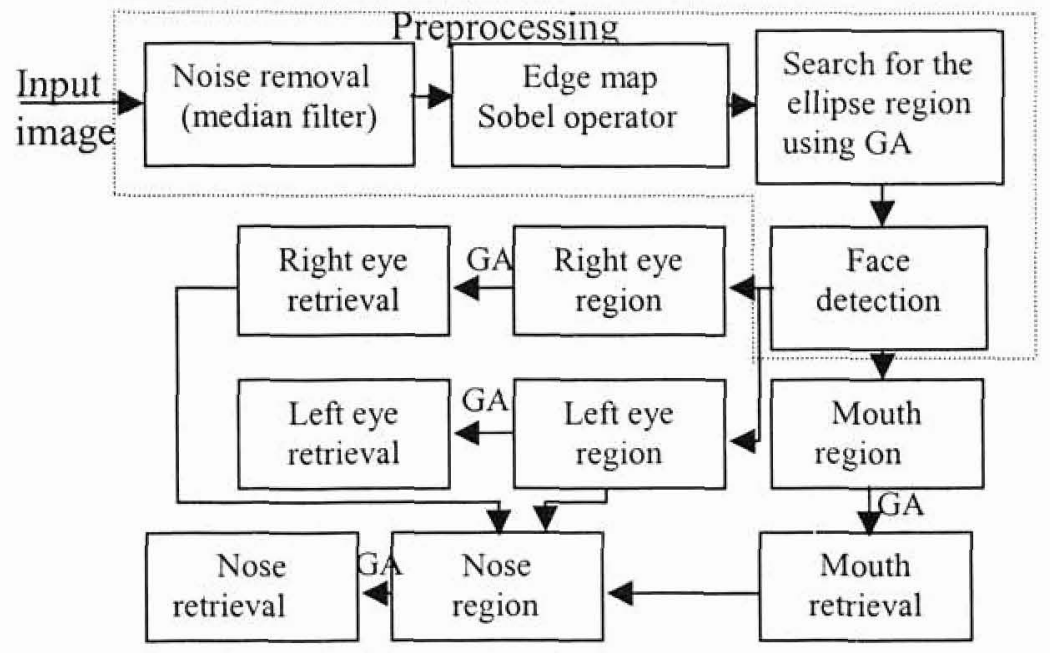

Figure 3.1 Block diagram of the proposed feature extraction method 


\subsection{Image acquisitions and noise removal}

All the images taken are the head and shoulder and frontal view of the person. They are originally stored in the color JPEG format. Matlab is used to convert the JPEG images into gray scale images. Smoothing filters are used for blurring and noise reduction. If the objective is to achieve noise reduction rather than blurring, median filters are used [45]. In this process the gray level of each of the pixel is replaced by the median of the gray levels in the neighborhood of the pixel [45]. This method is effective when the noise pattern consists of strong spike like components and the characteristic to be preserved is the edge sharpness, which is well suited for this application.

\subsection{Face segmentation using Genetic algorithm}

Two different approaches are used to detect the face region. The first one is based on the color information. This algorithm will fail to locate the exact faces if there are regions (hands and shoulders) other than the face that had similar color as the skin color present in the image. The second method is based on the assumption that the face region can be approximated by an ellipsoid [19]. This method is more robust compare to the earlier method. This method works well even under the environments when background is complex and the faces contain extra features such as spectacles, beard and etc. Locating faces in images containing skin color parts such as hands and shoulders poses no serious difficulty too. Thus this method has been adopted in this study to detect the facial area. 
The image captured initially is 1024 by 1536 . It is down sampled to 256 by 384 using the bilinear interpolation method The reduction in size will help to speed up the search process. The edge map of the image is then obtained using the 'Sobel' operator. The Sobel method detects edges using the Sobel approximation to the derivative. It returns edges at those points where the gradient of the image is maximum [45]. The mask of the Sobel algorithm is shown in Figure 3.2. The gradient of an image $\nabla f$ at location $(x, y)$ is defined as a vector,

$$
\nabla f=\left[\begin{array}{l}
G_{x} \\
G_{y}
\end{array}\right]=\left[\begin{array}{c}
\partial f / \partial x \\
\partial f / \partial y
\end{array}\right] .
$$

The magnitude of the vector is generally used for edge detection and is given by

$$
\nabla f=\left[G_{x}^{2}+G_{y}^{2}\right]^{1 / 2} .
$$

It is also common practice to use the gradient with absolute values, where,

$$
\nabla f=\left|G_{x}\right|+\left|G_{y}\right| .
$$

The masks below are used to find the partial derivatives $G_{\mathrm{x}}$ and $G_{\mathrm{y}}[45]$.

\begin{tabular}{|c|c|c|}
\hline-1 & -2 & -1 \\
\hline 0 & 0 & 0 \\
\hline 1 & 2 & 1 \\
\hline
\end{tabular}

(a)

\begin{tabular}{|l|l|l|}
\hline-1 & 0 & 1 \\
\hline-2 & 0 & 2 \\
\hline-1 & 0 & 1 \\
\hline
\end{tabular}

(b)

Figure 3.2 (a) Mask to calculate $G_{x}$ (b) Mask to calculate $G_{y}$ 
The ellipse can be represented by five parameters, the center $(x, y)$, the principal axis ( $A$ and $B$ ) and the orientation $\theta$. Genetic algorithm is used as a search tool to obtain the best match of ellipse region in the image. In locating the face region, the algorithm searches the ellipse region assuming that human face can be approximated by an ellipsoid. Initially the orientation of face to the principal axis was assumed to be zero. Each chromosome has four parameters, the center of the ellipse ( $x$ and $y), y$ directional radius $\left(r_{y}\right)$ and $x$ directional radius $\left(r_{x}\right)$. In the cases where the faces are oriented to either right or left side, orientation angle had to be incorporated in finding the face region. Therefore the chromosome should incorporate an additional parameter, the orientation angle, $\theta$.

The fitness of each individual in the population is defined by the ratio of the number of white pixels (edges) of the ellipse of the binary image to the actual number of white pixels that are suppose to be on the ellipse. The face is detected when the ratio of the number of edge pixels on the image $\left(N_{W}\right)$ to the number of pixels in the ellipse $(N)$ is high. Figure 3.3 shows the examples of imperfect and perfect match.

The fitness function $R$ is given as

$$
R=\frac{N_{W}}{N}
$$
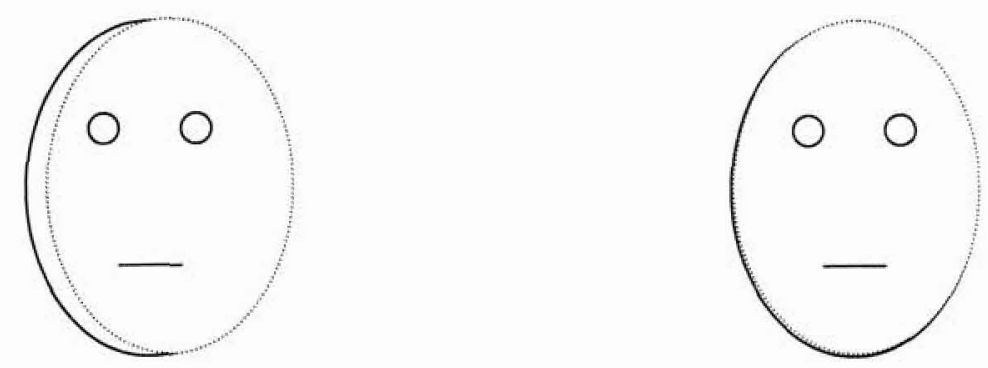

Figure 3.3 (a) Imperfect match and (b) perfect match 
It is obvious that $R$ is large when $N_{W}$ is large, and this occurs only when both the ellipses overlap perfectly (Figure 3.3(b)). When both the ellipses do not overlap perfectly (Figure 3.3(a) the value of $R$ will be low. It is assumed that the algorithm will ignore the faces that at located near the edges of the image. Also it is obvious that the length of the face is longer than the breath. That is $r_{y}>r_{x}$. This information will help us to reduce the size of the chromosomes and search space and at the same time speed up the search process. It is assumed that the ratio of the length to breath of the face is $1.5: 1$ and therefore the same ratio is used to obtain the face area once the ellipse region is located.

\subsection{Feature extraction using Genetic algorithm}

The located face region is mapped to the original image. The mapped faces are reduced to a size 300 by 200 so that all the faces are of the same size. Feature extraction is based on the edge density distribution. The horizontal edge map of the image from the face segmentation stage is obtained. The Sobel operator is again used for this purpose. The sizes of the templates for different features are decided according to general knowledge

of the size of the features. In this study we consider the size of the eye template is 51 by 71 , mouth is 41 by 101 and the nose is 21 by 61 . We use the edge density distribution in image space to extract the image. The feature areas will have high edge density than any other part in the face region. Figure 3.4 shows the distribution of edges in the horizontal map of the face region. 


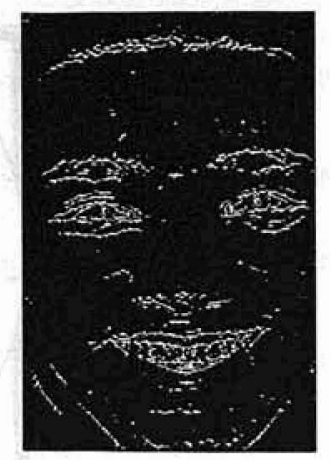

Figure 3.4 Edge map of the segmented image

It can be observed from Figure 3.4 that the edge density distribution around the feature point area is relatively high. To make the search process less computationally expensive, feature extraction can be carried out in different regions of the face. Basically, the face region can be divided into three sub regions as shown in Figure 3.5. The sub region $R_{r}$ is expected to contain right eye, the left eye is expected to be located in $R_{l}$ and the mouth in $R_{m}$. It is possible for the mouth region to contain nose. This problem can be easily overcome by evaluating the edge density projection of the mouth region. By analyzing the peak and valley, the mouth region can be identified. The nose region $\left(R_{n}\right)$ is then obtained once the eyes and mouth are located. The search region for nose is the region between eyes and mouth. 

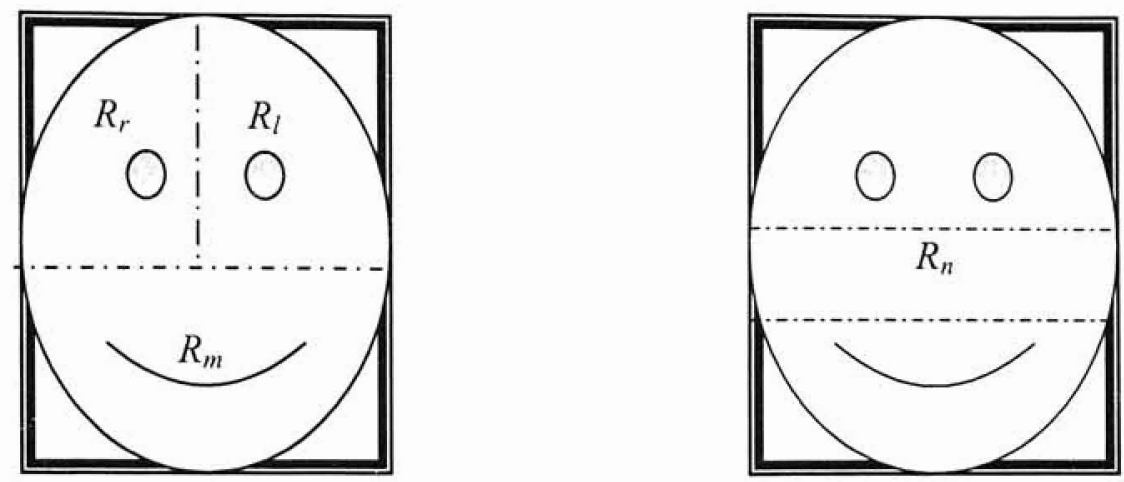

Figure 3.5 Sub regions of the face

The straightforward approach to find the elements with maximum fitness values is to search among all the elements and to compare the fitness values. In doing so the computational complexity is very high if the space size is large. To overcome this problem, GA is used as a search algorithm to locate the face region and the features.

In the feature extraction stage, GA is used to search the global maximum point when the template best matches the feature. The chromosome represents the position of the feature in the $x$ and $y$ direction. The fitness is evaluated in terms of density of the template. The best template is selected when the fitness is maximized.

The fitness function $F$ is shown below,

$$
F=\frac{1}{m \times n} \sum_{x=1}^{n} \sum_{y=1}^{m} T(x, y),
$$

where $\left\{\begin{array}{ll}T(x, y)=1 & \text { if the pixel is white } \\ \mathrm{T}(\mathrm{x}, \mathrm{y})=0 & \text { if the pixel is black }\end{array}\right.$, 
and $T$ is the template, $(x, y)$ are the coordinates of the template and $m \times n$ is the size of the template. Initially the population is chosen randomly. In each generation 20 percent of the population is considered for the reproduction. Roulette Wheel selection scheme is applied in the selection process. two-point crossover is applied by choosing the positions at random without duplication. Due to the crossover and mutation new individuals are introduced into the population. Insertion will help to introduce new individuals in the population, which cannot be produced due to crossover and mutation. A small percentage (i.e., one percent) of individuals are introduced in every generation.

GA helps in the case of feature extraction to search for the best matching template without matching all the points in the search space. 


\section{CHAPTER IV}

\section{SIMULATION STUDY AND RESULTS}

In this Chapter the simulation results of the proposed feature extraction approach is presented. A set of testing images was captured for the experiment. The images were taken with a Kodak Digital Science DC260 zoom camera with a resolution of 1024 by 1536 pixels. The data consist of more than 30 different head and shoulder images with at least two images per person. Some of the testing images contained multiple faces and faces oriented at an angle. There were no auxiliary lighting used when the images were captured. To be more specific, the images were captured in the natural lighting environment with no special background or equipment. To evaluate the robustness of the algorithm, images with different facial expressions, glasses and complex background were tested. Speckle noise was added to evaluate how robust the algorithm performs in the presence of noise. The size of the input images was 1024 by 1536 , and the size is reduced to 256 by 384 to reduce the computational complexity. The images used for the feature extraction are reduced to a size 300 by 200 for the purpose explained in Section 3.3. The edge map is obtained using the Sobel approximation with a threshold value of 0.02. The GA parameter setting used for both face segmentation and feature extraction in the simulation results are shown in Table 4.1. 
Table 4.1 GA Parameters

\begin{tabular}{||c|cc||}
\hline & $\begin{array}{c}\text { FACE } \\
\text { SEGMENTATION }\end{array}$ & $\begin{array}{c}\text { FEATURE } \\
\text { EXTRACTION }\end{array}$ \\
\hline Population Size & 100 & 50 \\
Crossover probability $\left(P_{c}\right)$ & 0.8 & 0.8 \\
Mutation Probability $\left(P_{m}\right)$ & 0.001 & 0.001 \\
\hline
\end{tabular}

The stopping criterion for face segmentation is 150 generations. In most cases, the faces were located in less than 100 generations. In the process of feature extraction, the stopping criterion was set to be 100 generations. Generally, the eyes and nose were located in less than 50 generations, the mouth was located in less than 75 generations.

Samples of the images captured by the camera in the original JPEG form are shown in Figure 4.1. These images are of size 1024 by 1536 . They are reduced to one quarter of their original size to reduce the computational workload. We are using Matlab function imresize, based on bilinear interpolation method to reduce the size of the original images. Here the output pixel values are calculated from a weighted average of pixels in the nearest 2-by-2 neighborhoods. This method is spatially more accurate, smother and less blocky (increase in visual quality) than nearest neighbor interpolation. 

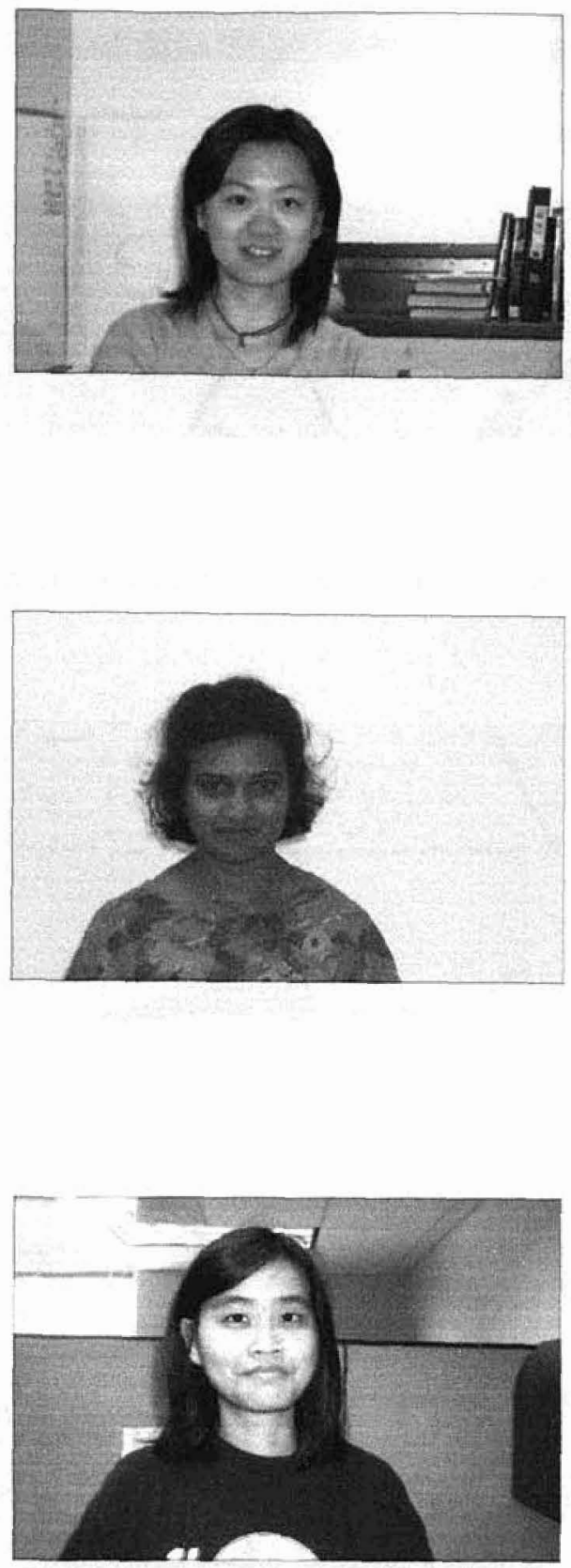

Figure 4.1 Color images captured by camera

The above images are converted to gray scale images. This operation is done so that they can be converted to binary images. The above images are converted to gray scale images 
using the matlab function rgb2gray. The corresponding gray scale images of Figure 4.1 are shown in Figure 4.2.
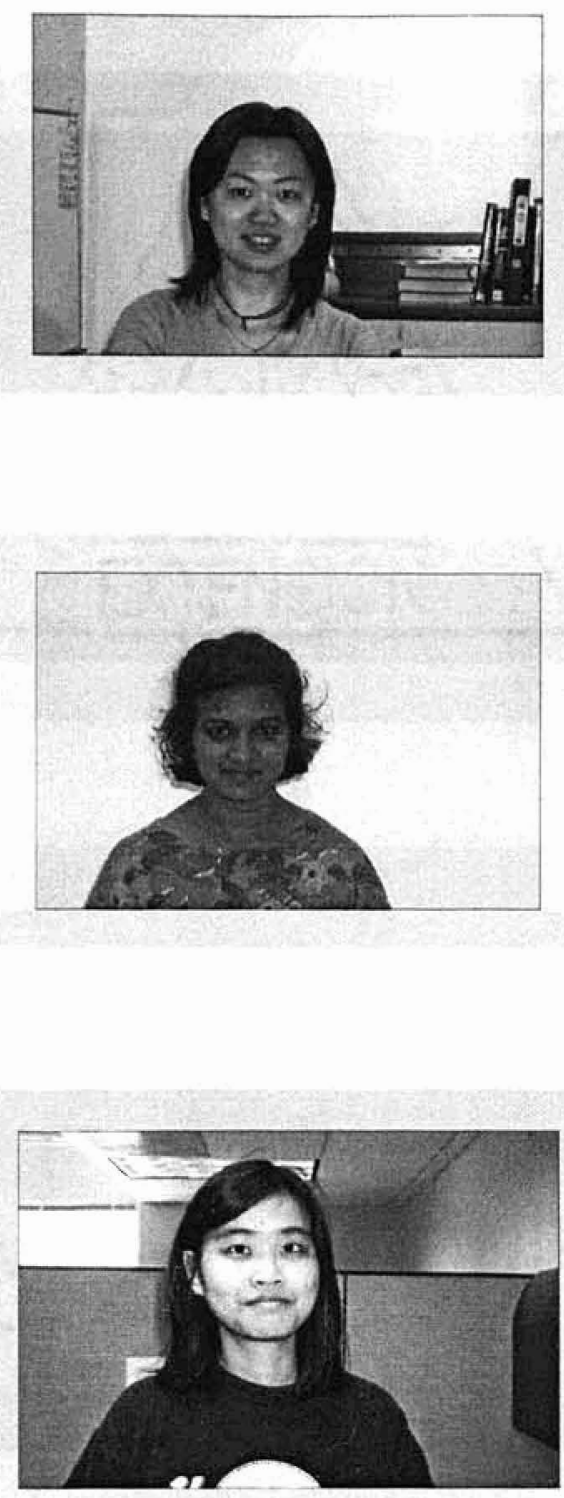

Figure 4.2 Gray scale images

The captured images may contain noise and the median filter is applied to remove the noise. Median filter will preserve the edge sharpness of the images. The image below 
shows the edge map of a gray scale image. The edge map of image is used to find the face location. It can be observed from Figure 4.3(a) and (b) that the face regions have almost the shape of ellipse. The algorithm used here will approximate the face region to the ellipsoid.

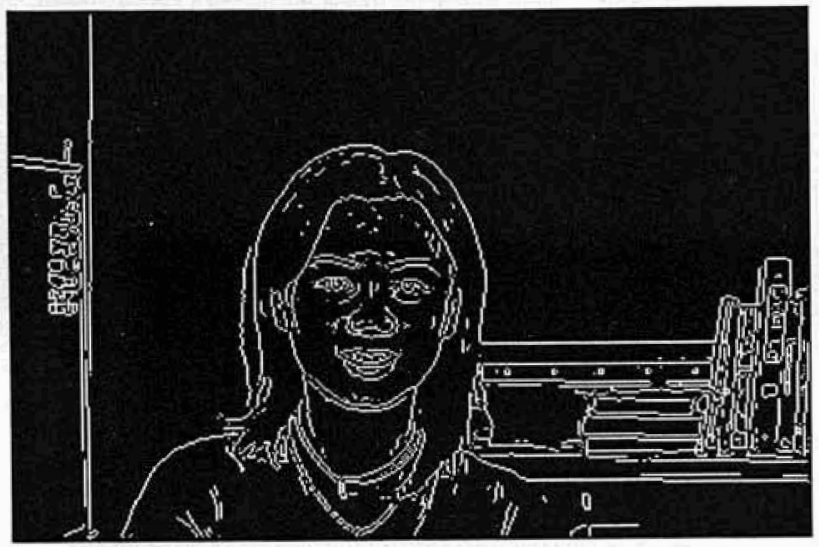

(a)

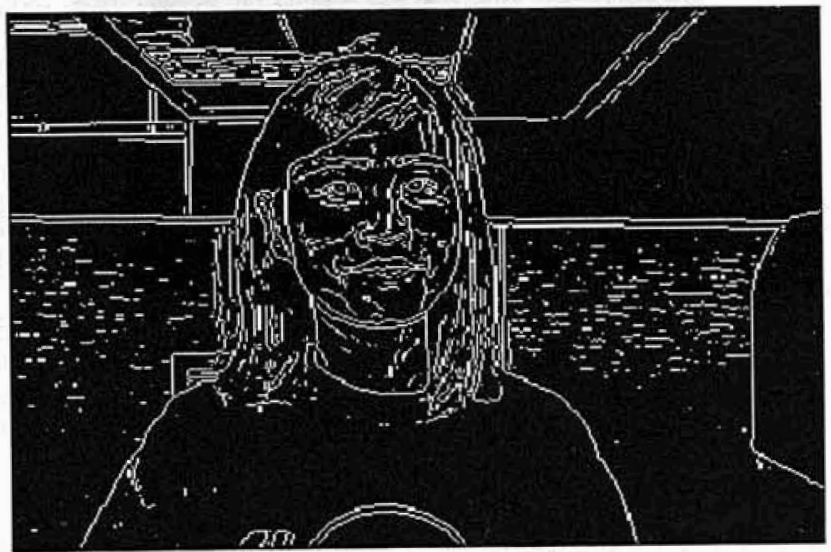

(b)

Figure 4.3 Edge map of the gray scale images 
Figures 4.4(a) and (b) shows the detected face regions. There are two lines at the bottom of the rectangle. Once the face region is detected, it is decided to store them in a particular ratio face so that feature extraction can be performed easily. In the experiments it is assumed that the face length / breadth is $1.5 / 1$. Thus one line is due to the ellipse detection and the other one is due to the ratio of face length / breadth assumption.

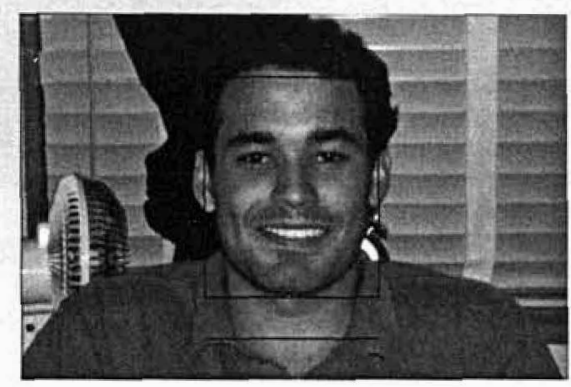

(a)

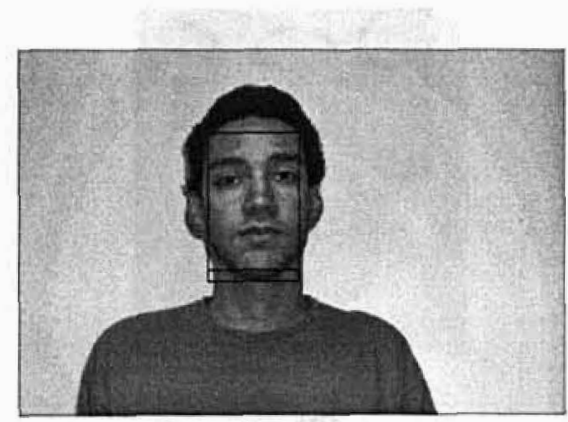

(b)

Figure 4.4 Face region detection

Figure 4.5 (a) shown below is a 256 by 384 image of the located face region. In most of the cases the faces are almost in the center of the image. There are no objects at the background. Therefore face segmentation becomes easy and was performed in less than 
50 generations. Figures 4.5 (b)-(e) are some of the simulation results showing the extracted facial features for the images taken in front of a plain background.

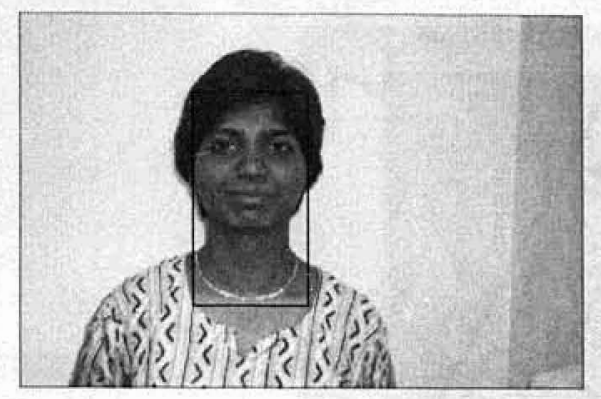

(a)

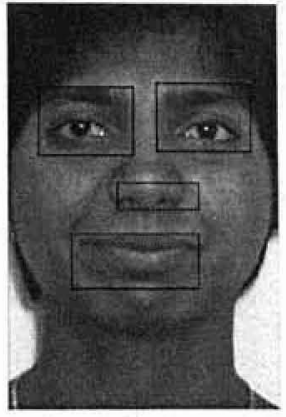

(b)

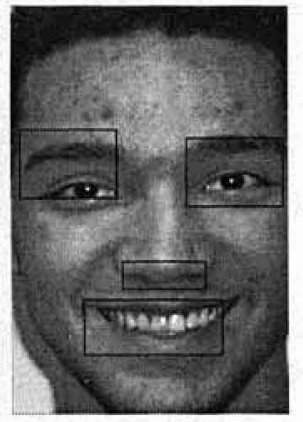

(c)

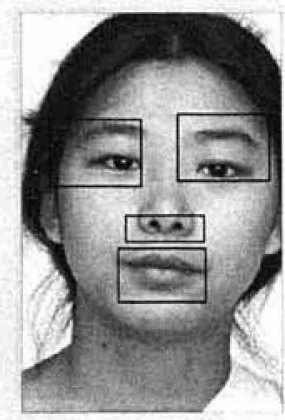

(d)

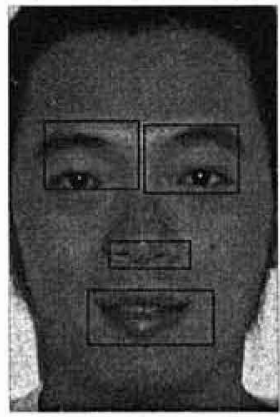

(e)

Figure 4.5 Simulation results of images with normal background

The images taken from complex background are shown in Figures 4.6(a), 4.7(a) and 4.8(a). A sample edge map of the image taken in complex background is shown in Figure 4.3(a) and (b). Unlike the previous case the edge distribution is more complex, thus face segmentation takes much longer time than those in the previous case. Figure 4.6(b) shows the extracted facial features from Figure 4.6(a). The images shown in Figure 4.7(b)-(e) 
are some examples of the images with various facial expressions. It can be observed from Figure 4.7(b) the mouth position has shifted from its nominal position and still it can be effectively extracted by the proposed algorithm.

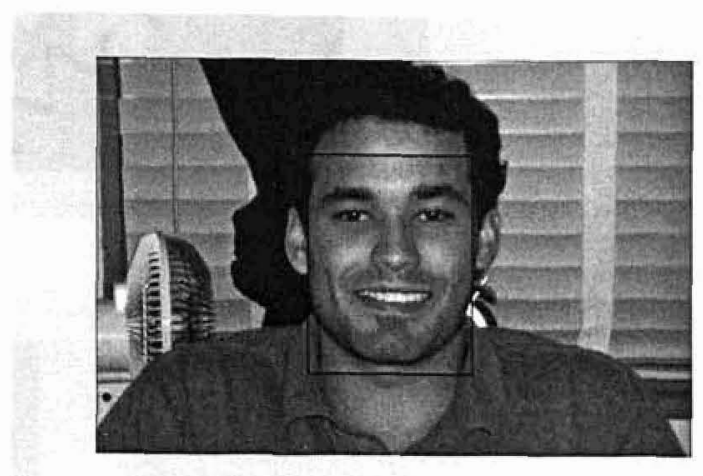

(a)

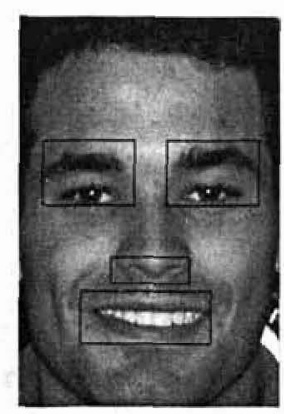

(b)

Figure 4.6 Simulation results of images with complex background

Once the features are extracted from the images, the rectangle windows of the features are adjusted so that they enclose the features perfectly. The edge map of each row of the template was obtained. A threshold value is used to get the final size of the mouth template. This can be observed well in Figure 4.7(b). 


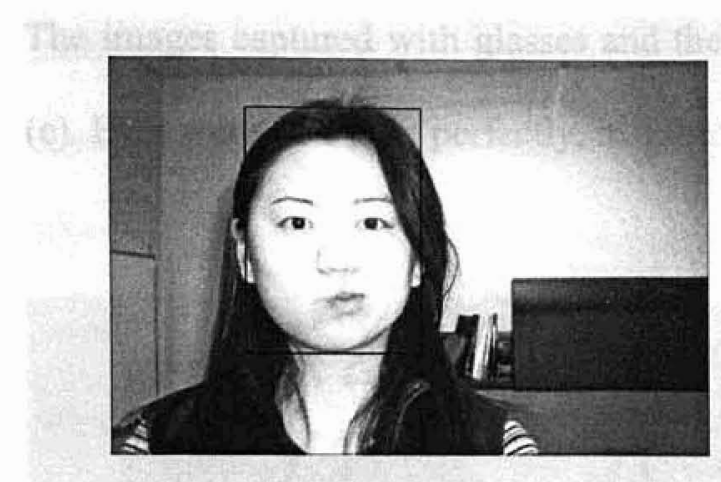

(a)

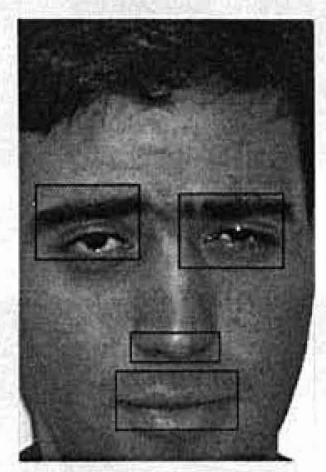

(c)

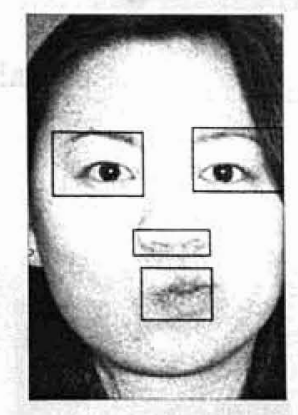

(b)

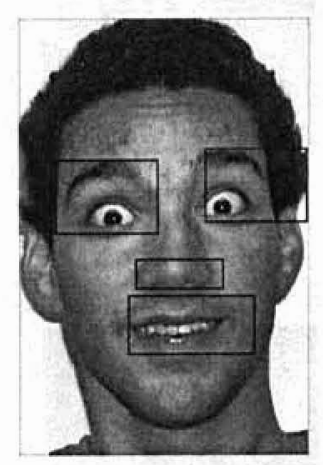

(e)

Figure 4.7 Simulation results of images with different expressions 
The images captured with glasses and the extracted features are shown in Figure 4.8(b)(e). Eyes were extracted perfectly, in spite of them being covered by glasses.

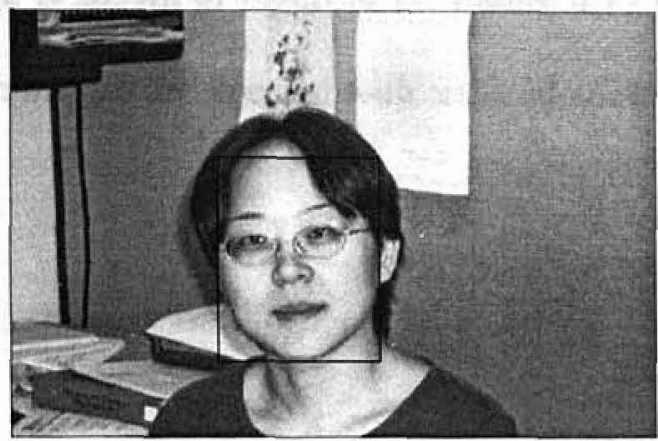

(a)

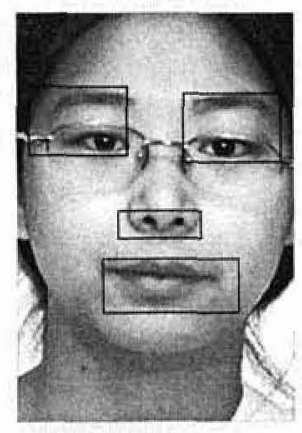

(c)

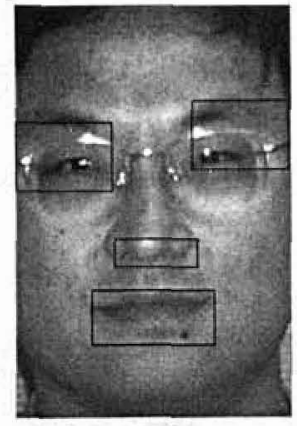

(d)

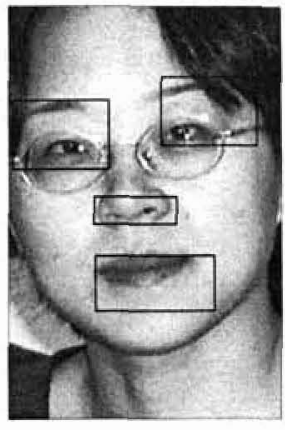

(b)

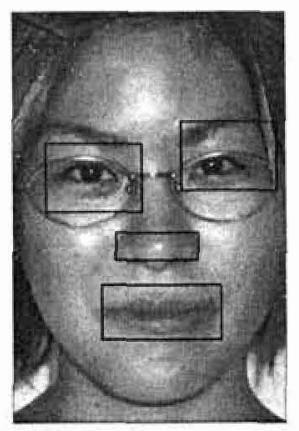

(e)

Figure 4.8 Simulation results of images with glasses

The robustness of the algorithm was tested when the images were injected with some artificial noise such as speckle noise. The speckle noise is a multiplicative noise. Noise corrupted image $(J)$ can be described by Equation (4.1).

$$
J=I+I \times n,
$$


The images captured with glasses and the extracted features are shown in Figure 4.8(b)(e). Eyes were extracted perfectly, in spite of them being covered by glasses.

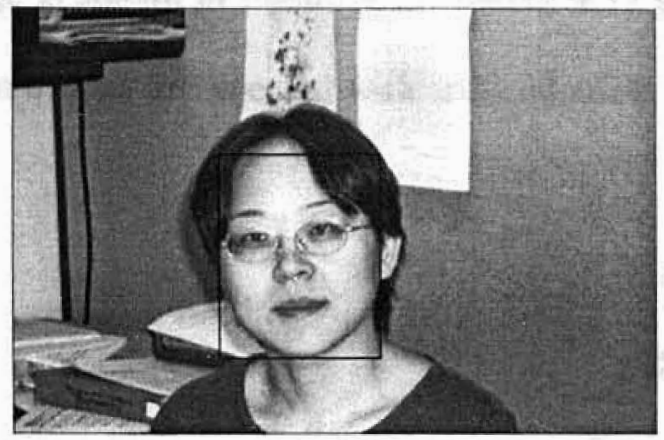

(a)

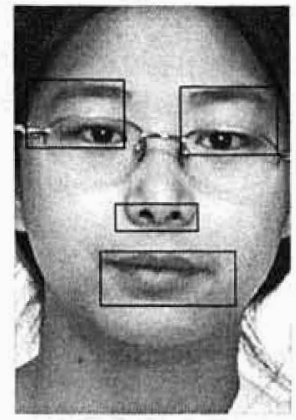

(c)

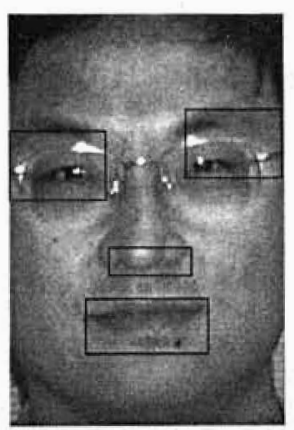

(d)

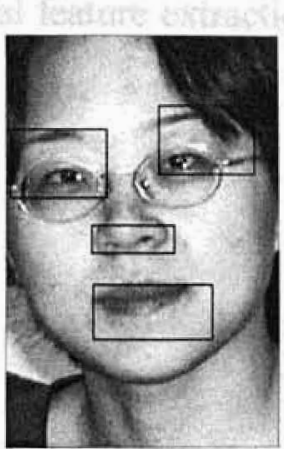

(b)

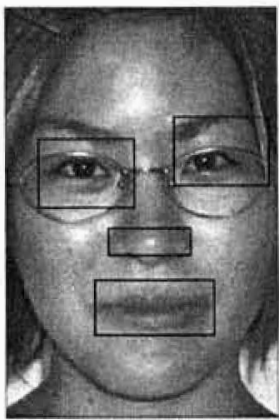

(e)

Figure 4.8 Simulation results of images with glasses

The robustness of the algorithm was tested when the images were injected with some artificial noise such as speckle noise. The speckle noise is a multiplicative noise. Noise corrupted image $(J)$ can be described by Equation (4.1).

$$
J=I+I \times n,
$$


where $I$ is the original image and $n$ is the uniformly distributed random noise with zero mean and $v$ variance. Images were tested with 0 mean and 0.02 variance. The results are shown below. Figure 4.9 shows the original image captured from the camera while Figure 4.10 is the image that was contaminated with noise. The edge map of the noise image is shown in Figure 4.11. Figure 4.12 shows the successful facial feature extraction when images are injected with artificial noise.

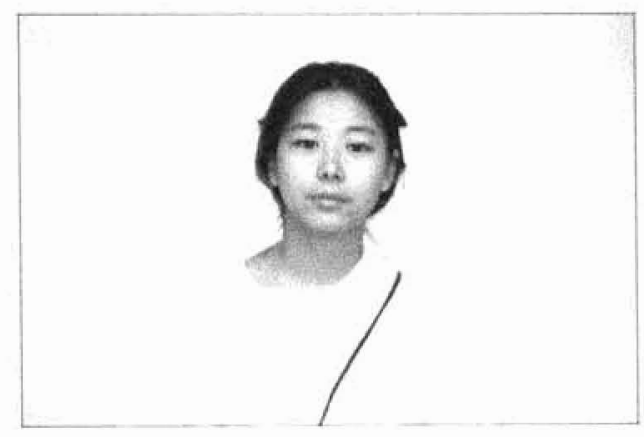

Figure 4.9 Image without noise

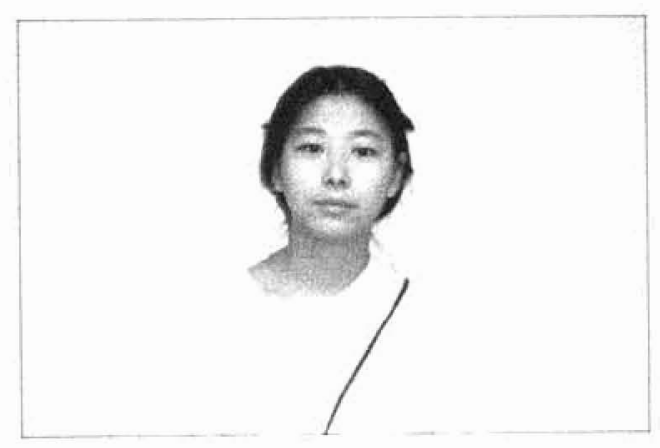

Figure 4.10 Image with noise 


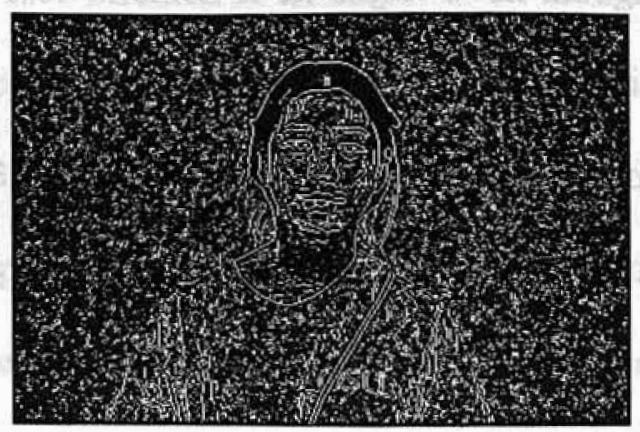

Figure 4.11 Edge map of the image containing noise

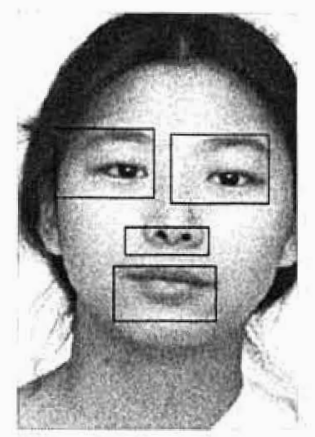

(a)

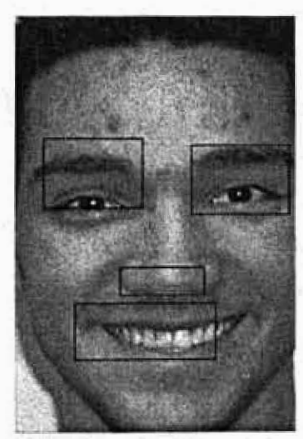

(c)

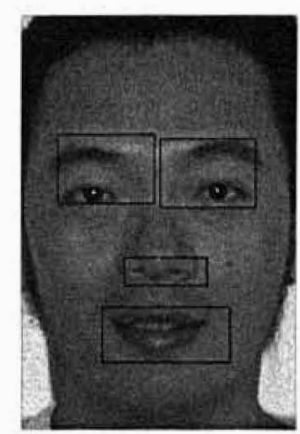

(b)

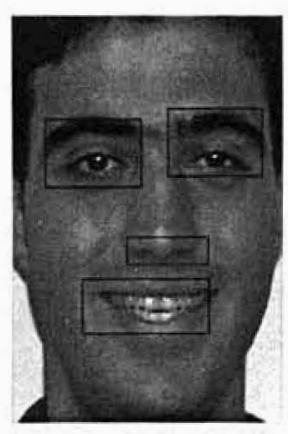

(c)

Figure 4.12 Simulation results of images with noise 
Figure 4.13(a) show the face oriented at an angle. Even though they can be segmented, the feature extraction becomes a difficult task. The face region cannot be divided as discussed in Section 3.3. Also the same template size cannot be used to extract the features. To avoid these problems, the detected face region is rotated to vertical position. The drawback in this method is that we may loose some information in the process of rotation. Figure 4.13(b) shows the results of feature extraction.

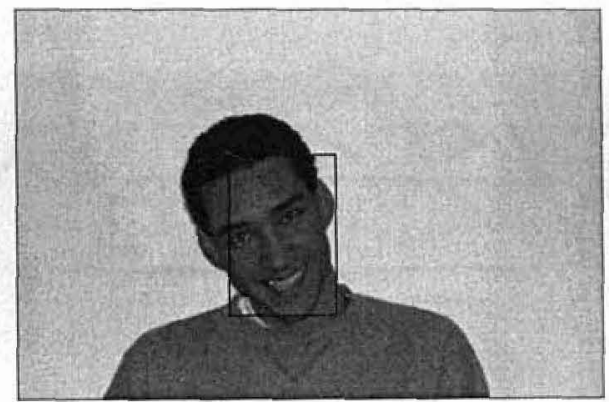

(a)

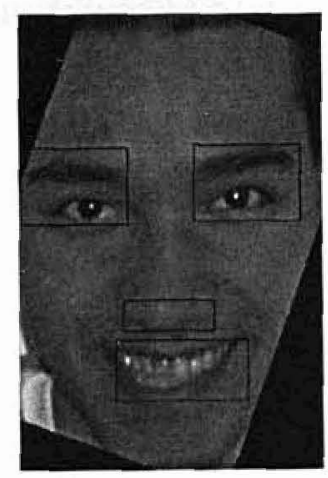

(b)

Figure 4.13 Simulation results of the images oriented at an angle 
More results were obtained with multiple faces in the images as shown in Figure 4.14(a) and Figure 4.15(a) and Figure 4.16(a). As there is more than one face in given images, searching was continued until a threshold is satisfied. The algorithm will be able to detect all the faces that can be approximated to an ellipsoid. The threshold value is based on the fitness value chosen. Also once the face is detected, it was made sure that the same face region is not selected again. This is achieved by assuming the fitness to be zero in the detected face regions in the succeeding search for additional face regions.

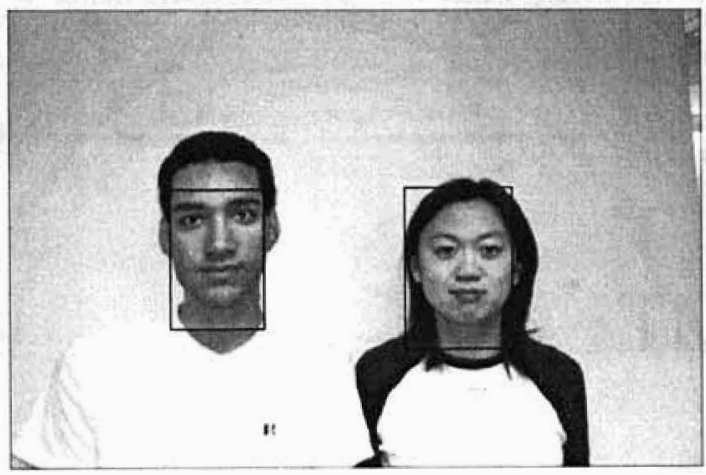

(a)

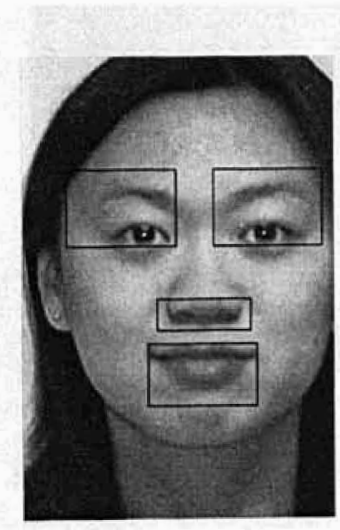

(b)

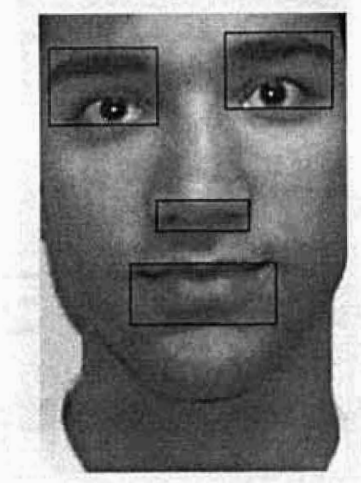

(c)

Figure 4.14 Simulation results of two faces in an image 
Figures 4.14(b)-(c), 4.15(b)-(c) and 4.16(b)-(d) show the simulation results of the facial feature extraction of multiple faces contained in a single image. The face on the right side of Figure 4.15(a) is oriented at an angle. In order to extract the features the face is rotated in the opposite direction. The effect is seen in Figure 4.15(c).

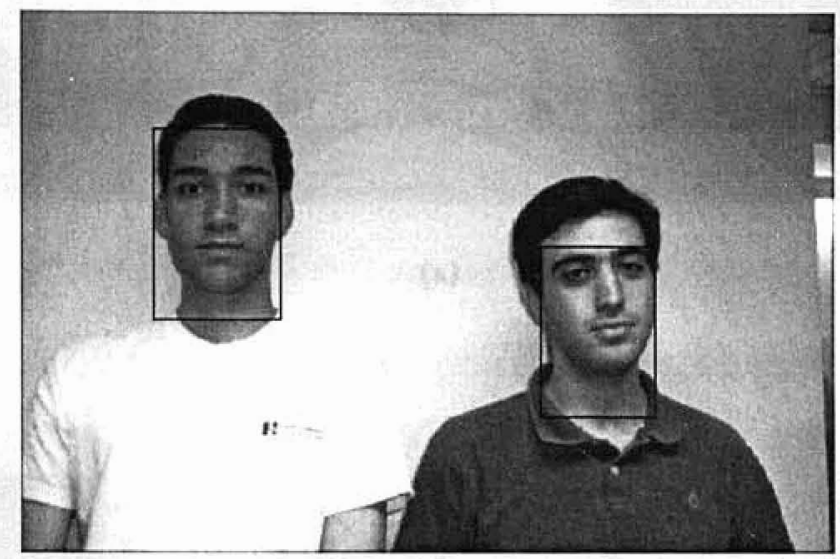

(a)

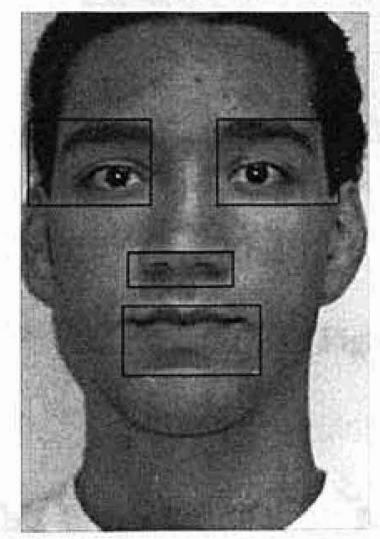

(b)

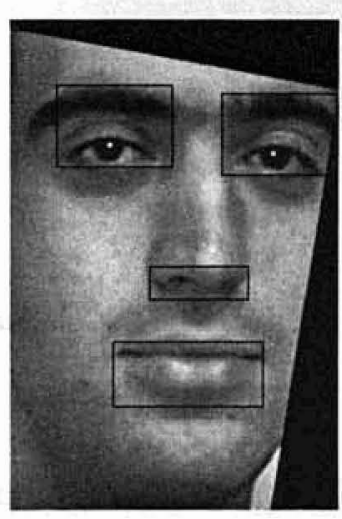

(c)

Figure 4.15 Simulation results of two faces in an image 


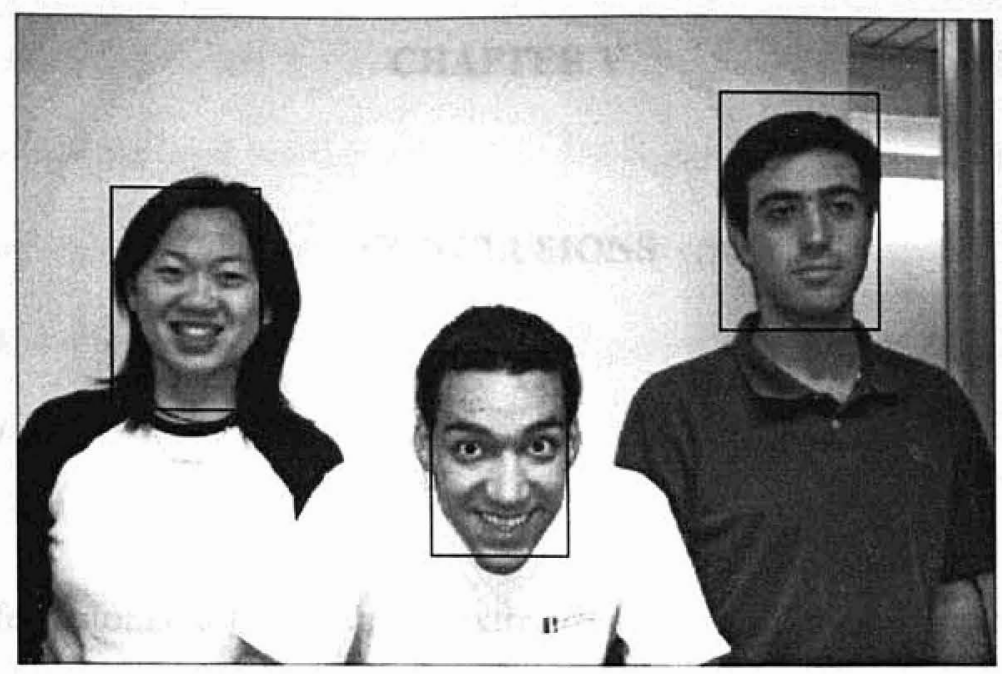

(a)

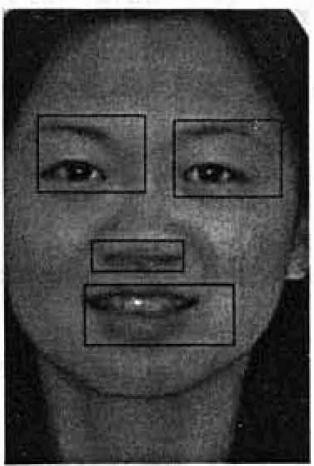

(b)

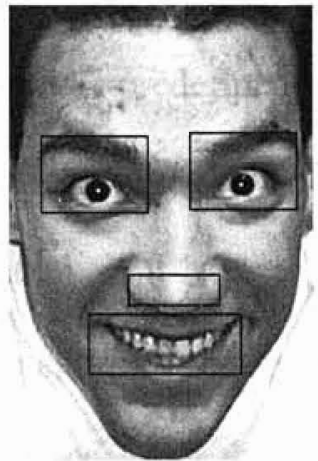

(c)

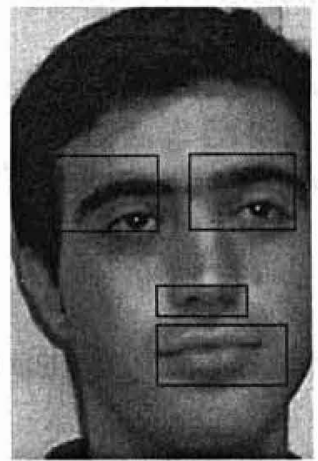

(d)

Figure 4.16 Simulation results of two faces in an image

The above simulation results demonstrate that the proposed feature extraction algorithm is able to retrieve the features effectively under various conditions. 


\section{CHAPTER V}

\section{CONCLUSIONS}

\subsection{Summary}

A procedure for automatic facial feature extraction from still images has been developed in this thesis. In order to extract the features from a head and shoulder image the face region is first located by a method that approximates the face to an ellipsoid. Genetic algorithm is used to search for the ellipse region. The facial features are extracted from a horizontal edge map of the image. Different sizes of templates are defined for different features and the features are searched in the predefined sub regions of the image. The cost function is based on edge density distribution. Facial features such as eyes, mouth and nose are searched using genetic algorithm.

The proposed facial feature extraction approach has been validated using a large number

of images. Some of the images contained more than one person, while others had the person oriented at an angle. Simulation results show that the facial features were extracted successfully.

Genetic algorithm was able to search effectively and reduce computational complexity, thus reducing the search time.

The template matching algorithms developed in [29] and [30] use different template patterns for matching which increases computational complexity, but the algorithm 
proposed in this study does not assume any specific template patterns for detection therefore the necessity to use too many different template patterns is avoided. Therefore the extraction process does not depend on the facial expressions or the resolution of the template. Also the cost function used is simple and easy to implement. The features were successfully extracted when the images contained glasses or different facial expressions. Some multiplicative noise was added to test the robustness of the algorithm. The algorithm was able to extract the features satisfactorily in most of the cases.

\subsection{Future Work}

The simulation results prove that the proposed algorithm is able to extract features successfully under different conditions. However, to extend this algorithm to some real world applications, it has to be improved further by incorporating the features outlined below:

- The algorithm can be enhanced further by adding more characteristics in the fitness function during the evolution process, so that the algorithm becomes more robust.

- The algorithm applied in this thesis was unable to detect features in the faces that contained either mustache or beard. Future work is needed in this area.

- Figure 5.1 shows a face that is not exactly in front view. These types of faces can be detected, though not accurately, but their features cannot be extracted using the proposed algorithm. Procedure must be developed to overcome this. 


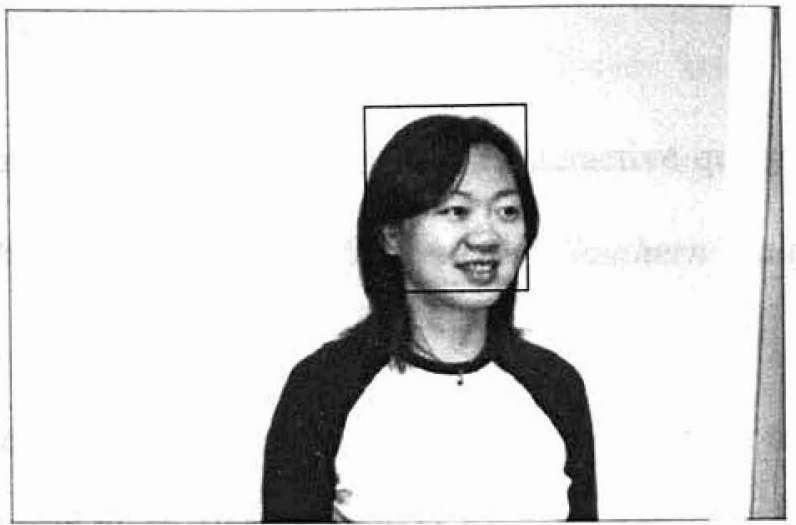

Figure 5.1 Face that is not exactly the front view 


\section{REFERENCES}

1. Wan, X., "Hierarchical feature extraction and interactive query for image data base management," Ph.D. Dissertation, University of Southern California, Los Angels, California, 1998.

2. Martin, C., "Development application of face recognition technologies for face recognition systems," Ph.D. Dissertation, University of Dayton, Dayton, Ohio.

3. Chellappa, R., Wilson, C.L. and Sirohey, S., "Human and machine recognition of faces: a survey," Proceedings of the IEEE, Vol. 83, No. 5, pp. $705-741,1995$.

4. Henry, V., "Biometrics: Face recognition technology," http://www.sans.org/infosecFAQ/authentic/face_rec.htm

5. Fitton, N. and Cox, S., "Linear feature extraction in geoscientific data," Proceedings of Digital Image Computing Techniques and Applications Conference, pp. 104-109, 1995.

6. Withworth, C.C., Duller, A.W.G., Jones, D.I. and Earp, G.K., "Aerial video inspection of overhead power lines," www.sees.bangor.ac.uk/dewi/otcs_web.htm

7. Li, H. and Chutatape, O., "Fundus image features extraction," Proceeding of the IEEE International Conference on Engineering in Medicine and Biology, pp. 3071$3073,2000$. 
8. Kovalerchuk, B., Conner, N., Ruiz, J. and Clayton, J., "Fuzzy logic for formalization breast imaging lexicon and feature extraction," www.azn.nl/rrng/xray/digmam/iwdm98/Abstracts/node51.html

9. Lin, C. and Ling W., "Automatic facial feature extraction by genetic algorithms," IEEE Transactions on Image Processing, Vol. 8, No. 6, pp. 834-845, 1999.

10. Welsh, W.J. and Shah, D., "Facial feature image coding using principle component," Electronic Letters, Vol. 28, No. 22, pp. 2066-2067, 1992.

11. Aizawa, K. and Huang, T.S., "Model based image coding: advanced video coding techniques for low bit-rate applications" Proceeding of the IEEE, Vol. 83, No. 2, pp. 259-271, 1995.

12. Chuang, M., Chang, R. and Huang, L., "Automatic facial feature extraction model based coding," Journal of Information Science and Engineering, Vol. 16, pp. 447458,2000 .

13. "America on alert Many Britons smile on spread of candid cameras," USA TODAY, September $26^{\text {th }} 2001$.

14. Daugman, J., "Face and gesture recognition: overview," IEEE Transactions on Pattern Analysis and Machine Intelligence, Vol. 19, No. 7, pp. 675-676, 1997.

15. "Facial Scan Technology: How it Works," http://facial-scan.com/facial-scan_technology.htm

16. Bhanu, B., Lee, S. and Ming, J., "Adaptive image segmentation using genetic algorithm," IEEE Transactions on Systems, Man and Cybernetics, Vol. 25, No. 12, pp. $1543-1567,1995$. 
17. Wang, Y. and Yuan, B., "Segmentation method for face detection in complex back ground," Electronic Letters, Vol. 36, No. 3, pp. 213-214, 2000.

18. Chai, D. and Ngan, K.N., "Locating facial region of a head-and-shoulders color image," Proceedings of the IEEE International Conference on Automatic Face and Gesture Recognition, pp. 124 -129, 1998.

19. Yokoo, Y. and Hagiwara, M., "Human face detection method using genetic algorithm," Proceedings of the IEEE Congress on Evolutionary Computation, pp. 113-118, 1996.

20. Brunelli, R. and Poggio, T., "Face recognition: features versus templates," IEEE Transactions on Pattern Analysis and Machine Intelligence, Vol. 15, No. 10, pp. 1042-1052, 1993.

21. Wu, H., Chen, Q. and Yachida, M., "Feature extraction and face verification," Proceedings of the IEEE International Conference on Pattern Recognition, pp. 484488, 1996.

22. Ham, Y.K., Lee, S.Y. and Park, R.H., "Knowledge based face recognition using neural networks," Proceedings of the International Joint Conference on Neural Networks, pp. 2125-2128, 1993.

23. Shah, D. and Marshall, S., "Statistical coding method for facial feature," IEE Proceeding of Visual Image Signal Processing, Vol. 145, No. 3, pp. 187-192, 1998.

24. Turk, M. and Pentland, A., "Eigenfaces for recognition," Journal of Cognitive Neuroscience, Vol. 3, No. 1, pp. 71-86, 1991. 
25. Turk, M.A. and Pentland, A.P., " Face recognition using eigenfaces," Proceedings of the IEEE Conference on Computer Vision and Pattern Recognition, pp. 586-591, 1991.

26. Peng, H., Rong, G and Bian, G., "Automatic face detection using adaptive subspace Method," Proceedings of the IEEE Conference on Systems, Man and Cybernetics, Vol. 1, pp. 88-92, 1996.

27. King, I. and $\mathrm{Xu}$, L., "Localized principal component analysis learning for face extraction and recognition," Proceedings of Workshop on 3D Computer Vision, pp. 124- 128, 1997.

28. "Photobook/eigenfaces demo," http://www-white.media.mit.edu/vismod/demos/facerec/basic.html

29. Baron, R.J., "Mechanisms of human facial recognition," International Journal on Man Machine Studies, Vol. 15, pp. 137-178, 1981.

30. Yagi, Y., "Facial feature extraction from frontal face image," Proceedings of the IEEE International Conference on Signal Processing, pp. 1225-1232, 2000.

31. Lau, C.M., Cham, W.K., Tsui, H.T. and Ngan, K.N., "An energy function for facials feature extraction," Proceedings of the International Symposium on Intelligent Multimedia, Video and Speech Processing, pp. 348-351, 2001.

32. Hines, H.L and Hutchinson, R.A., "Application of multi-layer perceptrons to facial feature location," Proceedings of the IEE International Conference on Image Processing and its Application, pp. 39-44, 1989. 
33. Hutchinson, R.A. and Welsh, W.J., "Comparison of neural networks and conventional techniques for feature location in facial images," Proceedings of the IEE International Conference on Artificial Neural Networks, pp. 201-205, 1989.

34. Yu, G., Liao, H.M. and Sheu, J., "A new fuzzy linear mapping technique for facial feature technique for facial feature extraction and recognition," Proceedings of the IEEE Conference on Neural Networks, pp. 1179-1184, 1996.

35. Mirhosseini, R. and Yan, H., "Neural networks for learning human facial features from labeled graph models," Proceedings of the IEEE Australian New Zealand Conference on Intelligent and Information Systems, pp. 170-173 1996.

36. Golgberg, D.E., Genetic Algorithm in Search, Optimization, and Machine Learning, Reading, M.A: Addison-Wesley, 1989.

37. Tang, K.S., Man, K.F., Kwong, S. and He, Q., "Genetic algorithms and their applications," IEEE Signal Processing Magazine, Vol. 13, No. 6, pp. 22-37, 1996.

38. Man, K.F., Tang, K.S. and Kwong, S., "Genetic algorithms: concepts and applications [in engineering design]," IEEE Transactions on Industrial Electronics, Vol.43, No. 5, pp. 519-534, 1996.

39. Mitchell, M., Introduction to Genetic algorithm, MIT Press Cambridge, Massachusetts, 1998.

40. Wong, K.W., Lam, K.M., Siu, W.C. and Tse, K.M., " Face segmentation and facial feature tracking for video applications," Proceedings of the IEEE International Symposium on Multimedia, Video and Speech processing, pp. 518-521, 2001. 
41. Huang, L.C. and Chen, C.W., "Human face feature extraction for face interpretation and recognition," Proceedings of the IEEE International Conference on Pattern Recognition, pp. 204-207.

42. Yuille, A.L., Cohen, D.S. and Hallinan, P.W., "Feature extraction from faces using deformable templates," Proceedings of the IEEE International Conference on Computer Vision and Pattern recognition, pp. 104-109, 1989.

43. Hjelmas, E.V., “ Biometric systems: A face recognition approach," Department of Informatics University of Oslo. w3.hig.no/ erikh/papers/nik2000.pdf

44. Wang, R. and Wang, Y., "Facial feature extraction and tracking in video sequence," Department of Electrical Engineering, Polytechnic University, Brooklyn, NewYork, USA.

vision.poly.edu:8080/ rwang/Papers/facial.doc

45. Gonzalae, R.C. and Woods, R.E., Digital image processing, Addison-Wesley, 1993.

46. Sabottka, K. and Pitas, I., "Face localization and facial feature extraction based on shape and color information," Proceedings of the IEEE Conference on Automatic Face and Gesture Recognition, pp. 483-486, 1996. 


\author{
VITA \\ Nethrie Nithianandan \\ Candidate for the Degree of \\ Master of Science
}

Thesis: FACIAL FEATURE EXTRACTION USING GENETIC ALGORITHM

Major Field: Electrical Engineering

Biographical:

Education: Bachelor of Science Degree in Electrical and Electronics Engineering from Karnataka Regional Engineering College, Surathkal, India, June 1999. Completed the requirements for the Master of Science Degree at Oklahoma State University in December 2001.

Experience: Graduate Research Assistant, Intelligent Systems \& Control Laboratory, Oklahoma State University, August 2000 to December 2001. Teacher Assistant, Oklahoma State University, August 2000 to May 2001.

Professional Membership: Associate Member of the Institution of Electrical Engineers, London. Student member, IEEE. 